\title{
Thermoelectric Power Factor: Enhancement Mechanisms and Strategies for Higher Performance Thermoelectric Materials
}

\author{
Arash Mehdizadeh Dehkordi ${ }^{1, a)}$, Mona Zebarjadi ${ }^{3}$, Jian $\mathrm{He}^{2}$, Terry M. Tritt ${ }^{1,2, a)}$ \\ ${ }^{1}$ Department of Materials Science and Engineering, Clemson University, Clemson, South Carolina 29634, USA \\ ${ }^{2}$ Department of Physics and Astronomy, Clemson University, Clemson, South Carolina 29634, USA \\ ${ }^{3}$ Department of Mechanical Engineering, Rutgers University, Piscataway, New Jersey 08854-8058, USA
}

Thermoelectric research has witnessed groundbreaking progress over the past 15-20 years. The thermoelectric figure of merit, ZT, a measure of the competition between electronic transport (i.e. power factor) and thermal transport (i.e. total thermal conductivity), has long surpassed once a longtime barrier of $\sim 1$ and thermoelectric scientists are targeting ZT $>2$ as the new goal. A majority of this recent improvement in ZT has been achieved through the reduction of lattice part of thermal conductivity $\left(\kappa_{l}\right)$ using nanostructuring techniques. The rapid progress in this direction focused the efforts on the development of experimental methods and understanding phonon transport to decrease lattice thermal conductivity. This fact left the development of ideas to improve electronic transport and thermoelectric power factor rather overlooked. With thermal conductivity of the potential thermoelectrics approaching the minimum theoretical limit, on the journey to higher ZT values, a paradigm shift is necessary toward the enhancement of the thermoelectric power factor. This article discusses the ideas and strategies proposed and developed in order to improve the thermoelectric power factor and thus hopefully move us closer to the target of a ZT > 2 ! 
1. Introduction

2. Best Electronic Band Structure

3. Thermoelectric Power Factor Enhancement Strategies

3.1. Strategies to Enhance Seebeck Coefficient

3.1.1. Modification of Density-of-States (DOS)

3.1.2. Resonant Levels

3.1.3. Hot Electron Filtering

3.2. Strategies to Enhance Electrical Conductivity

3.2.1. Modulation Doping

3.2.2. Crystallite Alignment

3.2.3. Composite Engineering

3.3. Strategies to Simultaneously Enhance Electrical Conductivity and Thermopower

3.3.1. Carrier Pocket Engineering and Convergence of Electronic Bands

3.3.2. Invisible Dopants

3.3.3. Interface Modification

4. Conclusions and Future Perspectives 


\section{Introduction}

The direct energy conversion between heat and electricity based on thermoelectric effects is a topic of long-standing interest in condensed matter materials science. Experimental and theoretical investigations in order to understand the mechanisms involved and to improve the materials properties and conversion efficiency have been ongoing for more than half a century. The potential of a material for thermoelectric (TE) applications, both power generation and refrigeration, is determined in general by a measure of material's dimensionless figure of merit, $\mathrm{ZT}$, defined as

$$
Z T=\frac{\alpha^{2} \sigma T}{\kappa}=\frac{P F}{\kappa}
$$

where $\alpha$ is the Seebeck coefficient, $\sigma$ the electrical conductivity, $\kappa$ the total thermal conductivity, and $T$ the absolute temperature in Kelvin. Figure of merit, in essence, is a measure of the competition between electronic transport (i.e. power factor, herein defined as $\left.P F=\alpha^{2} \sigma T\right)^{1}$ and thermal transport (i.e. total thermal conductivity) in a material.

For a long time, the progress had a slow pace and the best known thermoelectric materials were bismuth telluride-based alloys with a ZT around 1. However, since the renaissance of the thermoelectric materials research, which was started in early 1990s with the seminal paper of Hicks and Dresselhaus [1] that introduced the nanostructure concept as an improvement tool, groundbreaking progress has been achieved. The majority of the efforts to improve the ZT of thermoelectric materials have been focused in two main directions: (i) the reduction of lattice part of thermal conductivity by introducing rattler atoms in cage-like structures, embedding nanoparticles in the host matrix, or nanostructuring in more conventional materials, and (ii) the enhancement of the power factor. While groundbreaking success has been achieved through the former direction, progress has not developed as rapidly for the latter. With thermal conductivity of the potential thermoelectrics approaching the minimum theoretical limit, on the journey to higher ZT values, a paradigm shift is necessary toward the enhancement of thermoelectric power factor. There have been a number of excellent reviews on TE materials focusing on the progress in different classes of TE materials,[2,3] nanostructured thermoelectrics [4], and interfaces in bulk thermoelectrics.[5] There have also been excellent focused reviews on specific enhancement mechanisms to improve electronic transport.[6,7,8] Nevertheless, we believe that a place for a review on the power factor is missing. In this article, we aim to comprehensively review the mechanisms and approaches proposed in order to improve the thermoelectric power factor.

Figure 1 (a) and (b) show the temperature dependence of thermoelectric power factor reported for different classes of high performance $n$ - and $p$-type TE materials. It is observed that the majority of high performance thermoelectric materials possess power factor values between 0.8 to $2.5 \mathrm{~W} / \mathrm{m}-\mathrm{K}$. The largest power factor values reported in $n$-type materials belong to

\footnotetext{
${ }^{1}$ Traditionally, thermoelectric "power factor" is defined in the context of Z (not ZT) as $\alpha^{2} \sigma$ and has been used with the units of $\mu \mathrm{W} / \mathrm{cm}-\mathrm{K}^{2}$. However, defining the power factor as $\alpha^{2} \sigma \bar{T}$ brings about several advantages including (i) following SI units with the unit of $\mathrm{W} / \mathrm{m}-\mathrm{K}$, (ii) similar unit for the powder factor as that of thermal conductivity allows the researcher to easily compare different materials and readily estimate the figure of merit, ZT, knowing the thermal conductivity values, and (iii) it exhibits the true maximum potential of electronic transport in a material entailing the temperature dependent nature of properties. This refers to the fact that the maximum of $\sigma^{2} \sigma$ vs. T might happen at a different temperature than the maximum of $\alpha^{2} \sigma T$ vs. T plot that comes into ZT calculation.
} 
intermediate-valence $\mathrm{YbAl}_{3}$. Multiple filled skutterudites, half-Heusler alloys and $\mathrm{SiGe}$ are close seconds while they exhibit the largest reported values in $p$-type materials. Power factor values PF $\leq 0.8 \mathrm{~W} / \mathrm{m}-\mathrm{K}$ are reported for glass-like thermal conductivity materials (with $\kappa_{\mathrm{L}}<1 \mathrm{~W} / \mathrm{m}-\mathrm{K}$ ) such as Zintl phases, disordered $\mathrm{Zn}_{4} \mathrm{Sb}_{3}, \mathrm{La}_{3-\mathrm{x}} \mathrm{Te}_{4}$ and $\mathrm{SnSe}$ compounds. In order to be able to modify charge transport to our advantage to improve the thermoelectric power factor, we first need to understand the inherent materials properties. We start by briefly reviewing the main characteristics of good thermoelectrics, particularly favorable features of electronic band structure, which benefit the thermoelectric charge carrier transport and have been used traditionally as guidelines for materials selection. It should be noted that these are just general guiding principles that assist researchers in identifying new potential materials for TE applications. As we will see not all high-ZT materials follow these criteria. Due to the inherent trade-off between electrical conductivity and Seebeck coefficient, many of these selection rules are partially contradictory. In the second part of the article, we review the proposed enhancement mechanisms for power factor improvement.
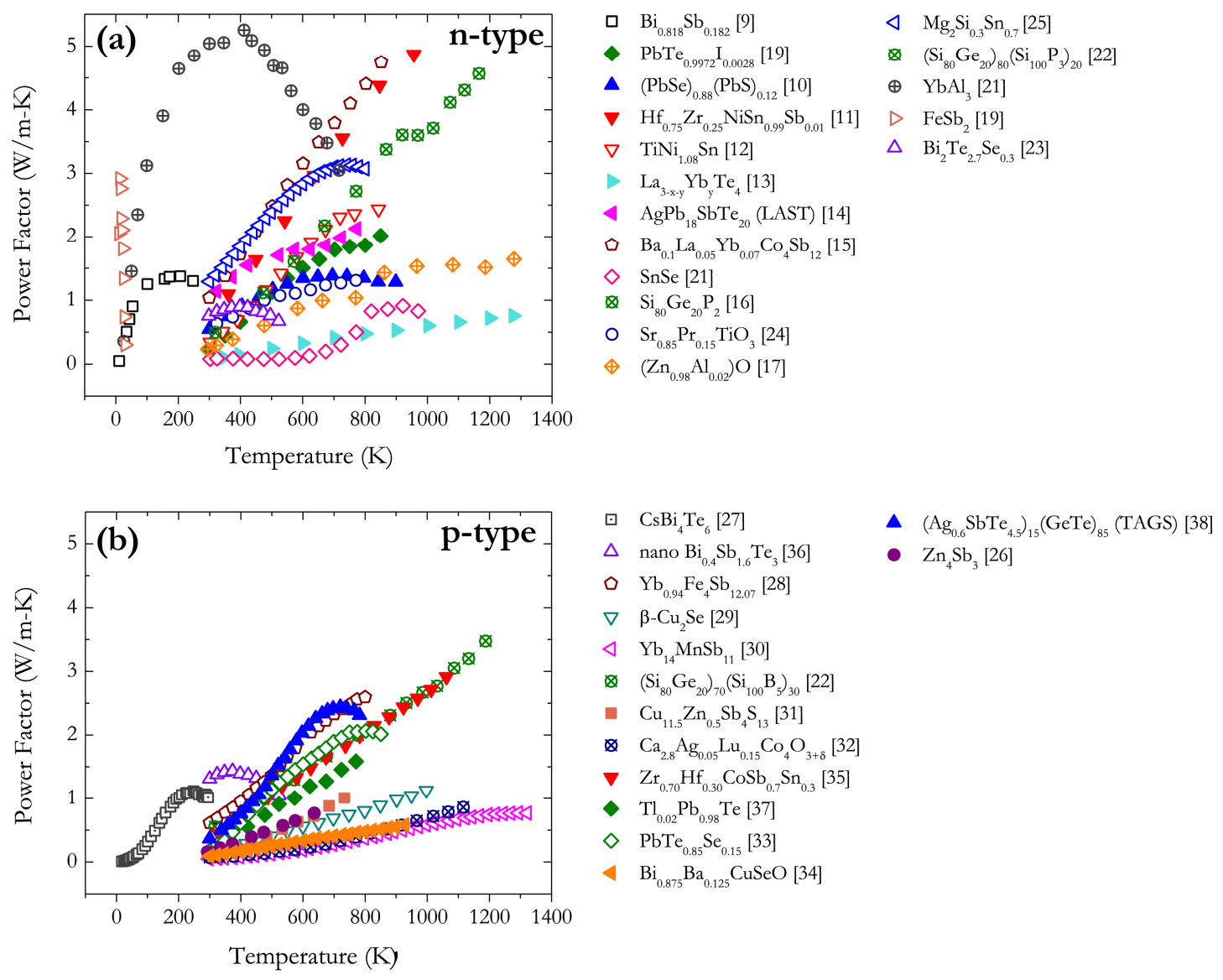

Figure 1- Temperature dependence of thermoelectric power factor, PF, reported for high-performance (a) $n$-type $[9,10,11,12,13,14,15,16,17,18,19,20,21,22,23,24,25]$ and (b) $p$-type $[22,26,27,28,29,30,31,32,33,34,35,36,37,38]$ thermoelectric materials. 


\section{Best Electronic Band Structure}

Ioffe's observation in doped semiconductors served as the first empirical attempt which highlighted the carrier concentration "sweet spot" of good thermoelectrics to be $n \sim 10^{18}-10^{20} \mathrm{~cm}^{-3}$, corresponding to degenerate semiconductors or semimetals. This follows the behavior of electronic transport in real-life materials as a function of carrier concentration. As the doping concentration increases, the electrical conductivity increases and the Seebeck coefficient decreases. Therefore there exists an optimum power factor versus doping concentration at relatively high concentrations and chemical potentials, $E_{F},{ }^{2}$ close to, or inside the conduction or valence band. The position of the optimum chemical potential depends on the type of the material and on the operating temperature. At room temperature, the optimum chemical potential for a low effective mass material such as GaAs is around 50meV[39] and for a heavy effective mass material such as silicon is around 200meV[40] above the bottom of the conduction band.

The intriguing question of "What is the best electronic band structure a thermoelectric material can have?" has been the subject of theoretical studies and experimental exploration for more than 30 years. The connection between band structure calculations and electronic transport coefficients is made via kinetic (or Boltzmann) transport theory. [41] This approach is valid for diffusive transport (i.e. dimensions larger than carrier mean free path) when the semiclassical picture is valid (i.e. mean free path larger than atomic distances and certain other generally very good approximations). The Bloch-Boltzmann expressions for electrical conductivity (along $x$ direction) and Seebeck coefficient are given by [42]

$$
\begin{gathered}
\sigma_{x}=e^{2} \int_{0}^{\infty} g(E) v_{x}^{2}(E) \tau(E, T)\left(-\frac{\partial f(E)}{\partial E}\right) d E_{x} \\
\alpha=\frac{k_{E}}{e}\left[\frac{\int_{0}^{\infty} g(E) v_{x}^{2}(E) \frac{E-E_{F}}{k_{E} T} \tau(E, T)\left(-\frac{\partial f(E)}{\partial E}\right) d E}{\int_{0}^{\infty} g(E) v_{x}^{2}(E) \tau(E, T)\left(-\frac{\partial f(E)}{\partial E}\right) d E}\right]
\end{gathered}
$$

where $e$ is the electron charge, $m^{*}$ the density-of-states effective mass, $\tau(E)$ the momentum relaxation time for charge carriers, $g(E)$ the total electronic density-of-states (DOS) and $f(E)$ the energy distribution function. The Fermi window factor $(-\partial f(E) / \partial E)$ is a bell-shaped

function centered at $E=E_{F}$ and has a width of $\sim k_{E} T$, where $k_{E}$ is the Boltzmann constant and $T$

the temperature. This factor is responsible for the fact that at a finite temperature only electrons near the Fermi surface contribute to the conduction process. It is observed that the Seebeck coefficient and electrical conductivity are connected through an energy-dependent function called the transport distribution function or differential conductivity, $\sigma(E)$, defined as

\footnotetext{
${ }^{2}$ To avoid confusion between the symbols for chemical potential and mobility, we use $\mu$ for carrier mobility and $E_{F}$ for chemical potential. $E_{F}$ is typically used for the Fermi energy, which is the chemical potential at $\mathrm{T}=0 \mathrm{~K}$.
} 


$$
\sigma(E) \equiv e^{2} g(E) v_{x}^{2}(E) \tau(E, T)
$$

$\sigma(E)$ is a measure of the contribution of electrons with energy $E$ to the total conductivity. It is observed that $\sigma(E)$ contain two types of energy-dependent component, (i) the band structure, which determines $g(E)$ and $v(E)$, and (ii) the scattering time $\tau(E, T)$, which is not directly determined by the band structure alone. If the chemical potential, $E_{F}$, is deep inside the band (degenerate limit, $E_{C}-E_{F}>k_{E} T$ ), i.e. for metals, the Seebeck coefficient in equation (3) could be approximated using Sommerfeld expansion and is referred to as the Mott formula,

$$
\alpha=\left.\frac{\pi^{2} k_{B}^{2} T}{3 e} \frac{1}{\sigma(E)} \frac{d \sigma(E)}{d E}\right|_{E=E_{F}}+O\left[T^{3}\right]
$$

Now the question is what band structure features maximize these expressions to yield the largest power factor values. The optimum band gap, $E_{g}$, of a thermoelectric semiconductor was first investigated theoretically by Chasmar and Stratton [43] for indirect gap semiconductors. They found the best gap of $6 k_{B} T$, where $T$ is the operating temperature of the thermoelectric device. Later Mahan [44] did a similar study and found $E_{g} \sim 10 k_{g} T$ to be a more realistic optimum for indirect gap materials. Sofo and Mahan [45] extended the calculations to include direct gap materials. They showed that the optimum gap is always greater than $6 k_{g} T$, but can be much larger depending on the dominant electron scattering mechanism. These calculations were in reasonable agreement with the experimental data on good thermoelectrics (e.g. $E_{g}=9.7 k_{B} T$ for $\mathrm{Bi}_{2} \mathrm{Te}_{3}$ at $300 \mathrm{~K}$ and $E_{g}=8.9 k_{B} T$ for $\mathrm{PbTe}$ at $650 \mathrm{~K}$ )[46] and have further validated the benefits of narrow-gap semiconductors for TE applications. Note that the band gaps reported in Ref. 46 were overestimated. The actual ratios are closer to $6 k_{E} T$ as will be shown later in this manuscript (see Figure 3). Nolas et al. [47] used single parabolic band model and optimized the position of the chemical potential for the single band model. They then added the bipolar contribution to the thermoelectric transport and calculated the modified reduced ZT. It was concluded that the bandgap should be larger than $8 k_{B} T$ in order to suppress the minority carriers' contributions to the thermoelectric transport. Figure 2 (a) and (b) demonstrate the idea of the optimum band gap for a simple semiconductor. Here, a simple semiconductor is defined as the one that could be described by one parabolic conduction band and one parabolic valence band and that the energy dependence of the scattering rates could be described by the same power law for both bands. Considering such a material, Seebeck coefficient is a function of only four parameters: the position of the chemical potential, the band gap, the energy exponent of the relaxation times $(r)$, and the ratio of the effective masses. 

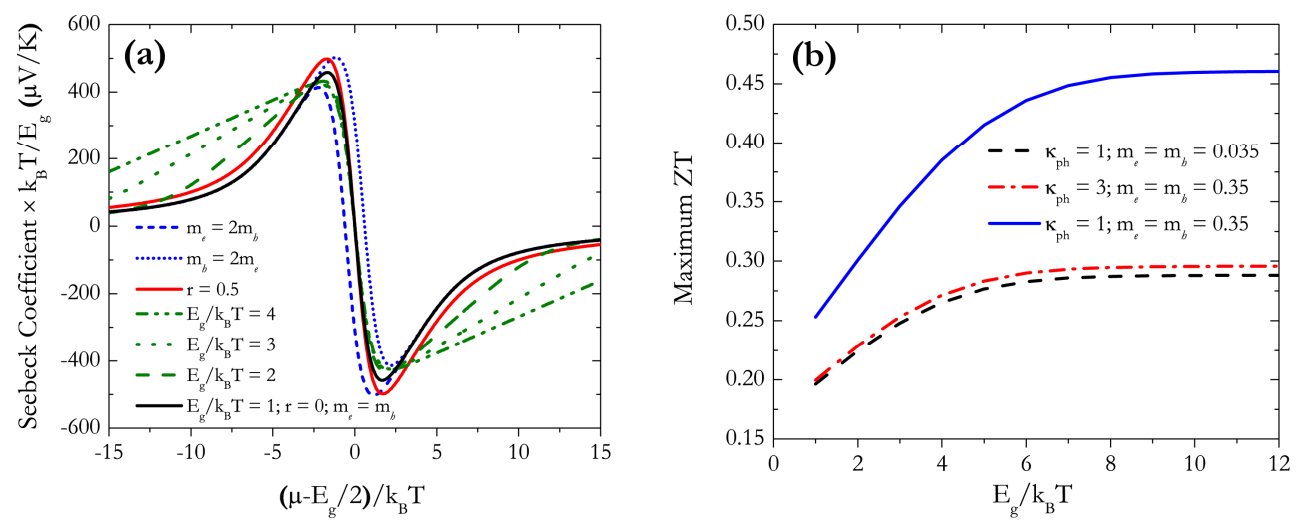

Figure 2 - (a) Seebeck coefficient calculated for two-band model. Two parabolic bands are considered, one for electrons and one for holes. Solid black line is the reference graph for which the parameters are set as listed in the legend: $\mathrm{E}_{\mathrm{g}} / \mathrm{k}_{\mathrm{B}} \mathrm{T}=1 ; \mathrm{r}=0 ; \mathrm{m}_{e}=$ $\mathrm{m}_{h}$. For each of the other graphs, only one parameter is modified and the rest of the parameters (which are not listed in the legend) are the same as the reference graph. (b) Maximum ZT calculated for the same two-band model. To calculate ZT, we assumed a constant phonon lattice thermal conductivity $\left(\mathrm{k}_{p h}\right)$ and we used constant relaxation time approximation $\left(\tau=10^{-13} \mathrm{~s}\right.$ for both bands). Calculations are done at room temperature. For a given band gap, ZT versus chemical potential was calculated and the maximum value of the corresponding curve is reported here.

This is illustrated in Figure 2 (a). As can be seen, a mass difference between electrons and holes would make the Seebeck coefficient slightly asymmetric. In other words, the Seebeck coefficient is slightly larger at the edge of corresponding heavier band and slightly smaller at the edge of the lighter band compared to bands with similar weights. The results are the same away from the band edges for all cases. Increasing the ' $r$ ' parameter increases the overall Seebeck coefficient in the entire chemical potential range. In Figure 2 (b), the maximum ZT values calculated for the two-band model, considering different parameters are plotted. In all cases, max ZT converges for $E_{g}$ values larger than $8 k_{B} T$, for smaller overall ZTs, the convergence is faster and happens at about $4 k_{B} T$. Today, we have observed that most of the good thermoelectrics possess narrow bandgaps and it is very hard to put a limit on the bandgap. Figure 3 (a) and (b) plot some of the best-reported thermoelectric power factor and ZT values versus the operating temperature. In order to compare with the bandgap, we have changed the temperature axis to $E_{g} / k_{g} T$ scale, where $E_{g}$ is estimated for each material from the reported values in literature. 

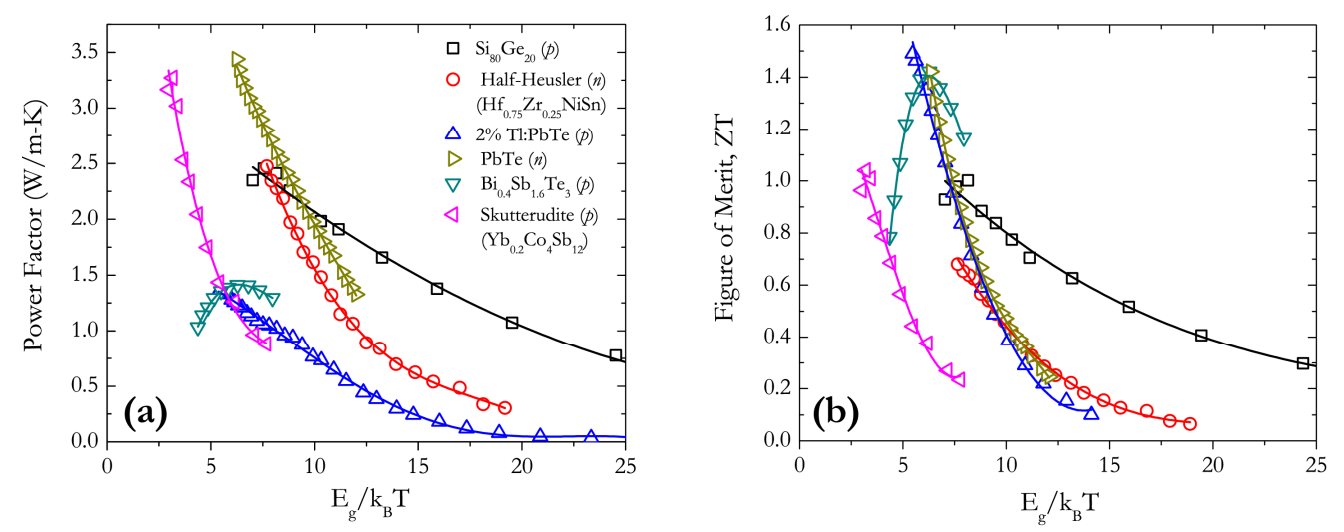

Figure 3 - (a) Thermoelectric power factor and (b) figure-of-merit for some of the best thermoelectric materials reported in the literature as a function of the band gap ( $p$-type $\mathrm{SiGe} \mathrm{[48],} p$-type $\mathrm{Bi}_{2} \mathrm{Te}_{3}$ [36], $p$-type PbTe [37], $n$-type PbTe [49], $n$-type half Heuslers [50], and $n$-type skutterudites [51]. Bandgap is estimated from the Ioffe website[52] for $\mathrm{Si}_{80} \mathrm{Ge}_{20}$ by linearly interpolating between $\mathrm{Si}$ and Ge and considering the temperature dependence of the bandgap, Similarly for $(\mathrm{BiSb})_{2} \mathrm{Te}_{3}$ we used interpolation of temperature dependent band gap of $\mathrm{Bi}_{2} \mathrm{Te}_{3}$ and $\mathrm{Sb}_{2} \mathrm{Te}_{3}$ taken from Springer database [53] , for PbTe we used a band gap of 0.36 after Ref. [ 54], for $\mathrm{Zr}_{0.25} \mathrm{Hf}_{0.75} \mathrm{NiSn} \mathrm{Eg}$ is taken to be $0.49 \mathrm{eV}$ by interpolating Ref. [55] data linearly, and finally for skutterudites we used Eg of 0.197eV from Ref. [51].

Another important feature of band structure, namely the density-of-states effective mass, $m^{*}$ and its close relationship with the carrier mobility, $\mu$, was first investigated in details by Goldsmid [56]. It was shown that the thermoelectric power factor, and hence ZT, are increasing functions of $\mu\left(m^{*} / m_{\theta}\right)^{3 / 2}$ (known as weighted mobility), where $m_{\circledast}$ is the free-electron mass. Therefore, ideally, it is desirable to maximize both $m^{*}$ and $\mu$. However, in practice, this is not quite simple due to their interplay.

It is known that larger effective mass materials correspond to larger Seebeck values, and therefore they are desirable for the thermoelectric applications. This dependence of the Seebeck coefficient to the effective mass is not clear from Equations (3) and (5) where the Seebeck coefficient is simply related to the position of the chemical potential and not to the effective masses. However, the position of the chemical potential, for a fixed doping concentration, depends on the materials effective masses through their density of states, $g(E)$. Therefore, if the Seebeck coefficient is plotted versus the carrier concentration (so-called Pisarenko plot), it is expected to observe larger Seebeck values for larger effective masses. The Seebeck coefficient versus carrier concentration is plotted for some of the good thermoelectric materials in Figure 4. 


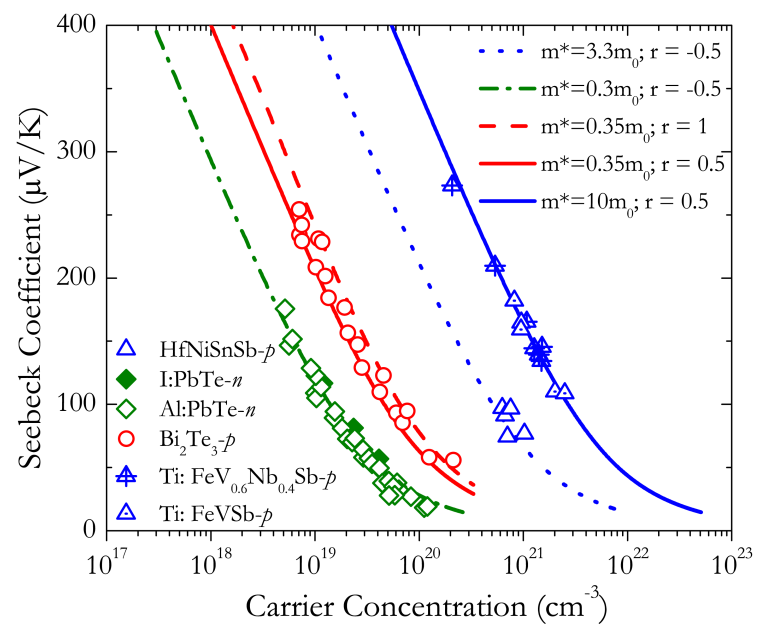

Figure 4 - Seebeck coefficient versus carrier density for some of the good TE materials that could be explained using single parabolic band model. Data are taken from [36] for $p$-type $\mathrm{Bi}_{2} \mathrm{Te}_{3}$, [19] for I-doped PbTe, [57] for Al-doped PbTe, and for $p$-type half-Heuslers from [58] for $\left(\mathrm{Hf}_{0.6} \mathrm{Zr}_{0.4}\right)_{1-\mathrm{x}}(\mathrm{Yb}, \mathrm{Sb})_{\mathrm{x}} \mathrm{NiSn}_{0.98} \mathrm{Sb}_{0.02}$, [59] for Ti-doped FeVSb, and [60] for Ti-doped $\mathrm{FeV}_{0.6} \mathrm{Nb}_{0.4} \mathrm{Sb}_{\text {. }}$

As explained above and can be observed in Figure 4, in general, materials with larger effective masses possess larger Seebeck coefficients. However, heavy carriers are less mobile $\left(\mu=\tau=\tau / m^{*}\right)$ and so again the trade-off appears. Figure 5 shows the reported Hall mobility values as a function of carrier concentration for different classes of high-performance $n$ - and $p$ type thermoelectric materials. It is observed that for highly doped TE materials with $\mathrm{n} \geq 10^{20}$ $\mathrm{cm}^{-3}$, mobility values of $\leq 100 \mathrm{~cm}^{2} / \mathrm{Vs}$ is reported. The highest values are reported for PbTebased thermoelectrics for both $n$ - and $p$-type, which have relatively low effective masses. 
Figure 5 - Room temperature Hall mobility as a function of carrier concentration for high performance $n$-type thermoelectric materials $[10,13,14,15,22,24,49,61,62,63,64] \quad$ (a) and p-type thermoelectric materials $[28,29,35,37,63,64,65,66,67,68,69,70,71,72,73]$ (b). Filled symbols represent values reported for single crystals (annotated in the legends with "single").

During the years several ideas were proposed to suppress the interplay between electrical conductivity and Seebeck coefficient and provided us with several insights on ideal crystal and band structure, which have served as materials selection criteria. Mahan [46] showed that in order to increase the density-of-states effective mass without sacrificing the mobility very much, the multivalley semiconductors with small inertial masses are desirable. For the most general case, the density-of-states effective mass is equal to $N_{v}^{2 / 3}\left(m_{1} m_{2} m_{3}\right)^{1 / 3}$, where $N_{v}$ is the number of equivalent valleys (band degeneracy) and $m_{1,2,3}$ are inertial masses in each direction. Provided that intervalley scattering is minimized or absent, theory suggests that a multivalley semiconductor with small inertial masses would have the largest value of $\mu\left(\mathrm{m}^{*} / \mathrm{m}_{\mathrm{g}}\right)^{3 / 2}$. This maximizes the thermoelectric power factor particularly through optimization of Seebeck coefficient over that of the single-valley counterpart. For fixed $E_{F}$, multivalley band structure yields the same Fermi velocities but higher $g\left(E_{F}\right)$, and therefore larger carrier density $n(E)$, but the same Seebeck coefficent. Mahan $[46,44]$ found the presence of at least four band minima is necessary for good thermoelectrics. The number of equivalent conduction valleys for $\mathrm{Bi}_{2} \mathrm{Te}_{3}$, $\mathrm{PbTe}$, and $\mathrm{SiGe}$ are 6, 4, and 6, respectively (excluding the spin degeneracy).[6,46] Compounds with a large number of equivalent valleys are typically those with high symmetry crystal 
structures.[74] Figure 6 schematically compares the multivalley conduction band of $\mathrm{Bi}_{2} \mathrm{Te}_{3}$ with that of GaAs.
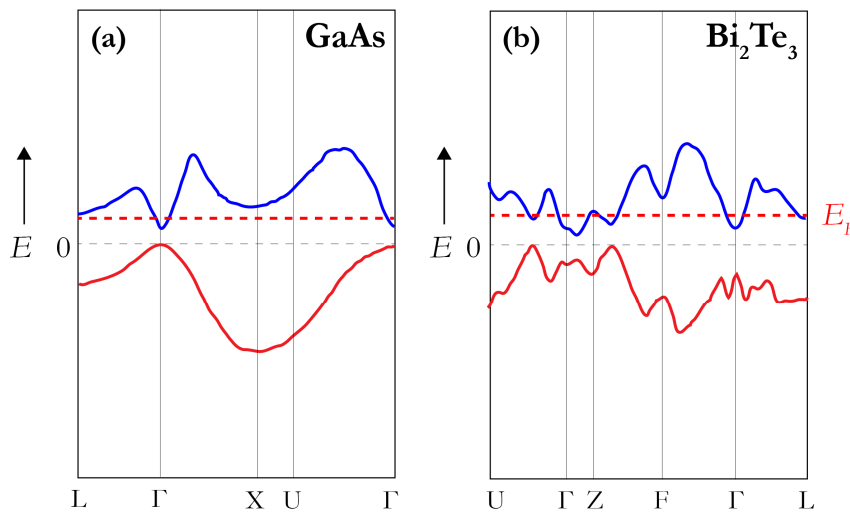

Figure 6 - Schematic band structure of (a) GaAs [75] with a single conduction valley, [46] and (b) $\mathrm{Bi}_{2} \mathrm{Te}_{3}$ [76] with 6 equivalent conduction valleys.

Another idea is to take advantage of the anisotropic band masses. Anisotropic band masses allow high average Fermi velocities due to the light mass directions(s) (so high plasma frequencies and electrical conductivity) at the same time as strong logarithmic derivatives. This idea ties in with the idea of using multivalley band structures. In order to maximize the weighted mobility, while high mobility along the current flow direction is needed, high effective masses need not occur along the same crystallographic directions. Therefore, there should be good opportunities to optimize highly anisotropic structures. Carrier mobility should be high (small effective mass) along the direction of transport while there are many states (heavy mass) available in the transverse direction.[77] A recent report on high ZT of 2.6 along one crystallographic direction of the high-temperature phase in SnSe, illustrates the potential of anisotropic materials.[20] Although the high ZT is originated from ultralow thermal conductivity, which is attributed to high anharmonicity of the chemical bonds, the electronic transport is still interesting. These single crystal samples possess 8 times larger room-temperature carrier mobility along b-axis versus a-axis (for similar carrier concentration and Seebeck coefficient values). This leads to a 5fold larger power factor in the direction of b-axis. Of course, this result needs to be duplicated by other researchers.

Electronegativity difference, $\langle\Delta \chi\rangle$, among constituent elements of a thermoelectric material (e.g. $\langle\Delta \gamma\rangle=0.3$ for $\mathrm{Bi}_{2} \mathrm{Te}_{3}$ and 0.53 for $\mathrm{PbTe}$ ) was also found to be an effective screening parameter for high carrier mobility materials, which was proposed by Slack. [78] Slack pointed out that materials with small electronegativity difference among their constituent atoms have high mobility values. $\langle\Delta \chi\rangle \leq 0.5$ has been proposed as an initial screening value in the search for good thermoelectric materials. Skutterudites were first identified as such by Slack, on the basis of this screening process $\left(\langle\Delta \gamma\rangle=0.11\right.$ for $\left.\mathrm{CoSb}_{3}\right)$. Electronegativity difference is a measure of the covalency of the bonding in a material. Large $\langle\Delta x\rangle$ indicates ionic bonding and the scattering of carriers by polar optical phonons which reduce the carrier mobility significantly.[78] This is one of the reasons oxides were believed to make poor thermoelectrics (e.g. $\langle\Delta \alpha\rangle=1.79$ for $\mathrm{ZnO}$ and 2.2 for $\mathrm{SrTiO}_{3}$ ). 
As it is seen in equations (2) and (3), another band structure parameter which plays a crucial role in the electronic transport is the density-of-states of charge carriers. Mahan and Sofo [79] treated the problem of "What is the best electronic structure a thermoelectric can have?" as a problem in mathematics. They showed that the transport distribution function (or differential conductivity), $g(E) v_{\alpha}^{2}(E) \tau(E)$, that maximizes the power factor is delta function-shaped. Since the exact condition is not found in nature, they proposed the search for materials with narrow energy distribution of carriers but with high carrier velocity in the direction of transport. Optimal density-of-states for thermoelectric applications was suggested to possess sharp singularity in the vicinity of the chemical potential. The nature's closest approximation to delta function is observed in $f$-level rare-earth compounds.[80] $\mathrm{YbAl}_{3}$ and $\mathrm{CeSn}_{3}$ are examples of such materials possessing the highest ever reported values of power factor of 5.4 and $3.0 \mathrm{~W} / \mathrm{m}-\mathrm{K}$ at $300 \mathrm{~K}$. [21,46] Figure 7 schematically compares the sharp-featured density-of-states of $\mathrm{YbAl}_{3}$ [81] to that of the GaAs. It was suggested, but not proven, that this behavior holds true also for $3 d$ electrons in $\mathrm{FeSb}_{2}$.[82]
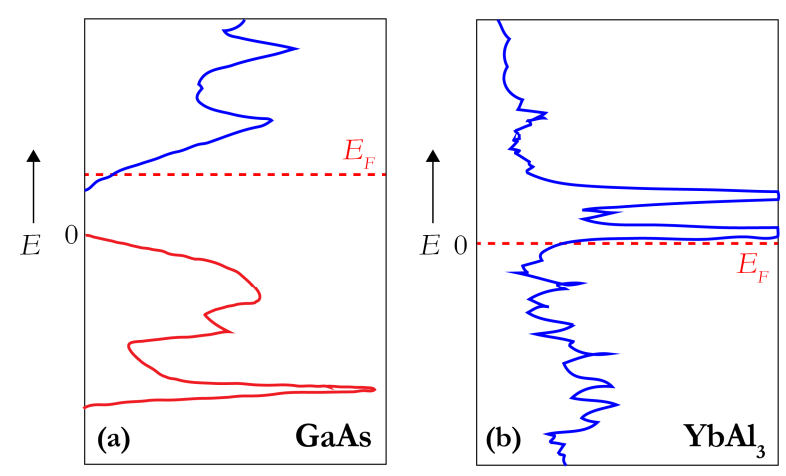

Figure 7 - Schematic density-of-states of (a) GaAs [83] and (b) $\mathrm{YbAl}_{3}[84,85]$ possessing sharp features close to Fermi level.

Recently, Zhou et al. [86] reinvestigated Mahan and Sofo's theory by considering the scattering model of carriers. They concluded that when the bandwidth is zero, the transport distribution function, $g(E) v_{x}^{2}(E) \tau(E)$, is finite and not infinite as previously speculated by Mahan and Sofo, even though the carrier density of states goes to infinity. Such a finite distribution of energy of carriers results in a zero electrical conductivity and thus a zero ZT. Therefore, there exists an optimal electronic bandwidth which depends highly on the carrier scattering models. Their calculations show that the optimal ZT cannot be found in an extremely narrow conduction band. Jeong and Lundstrom [87,88] have also recently revisited the "best bandstructure question" in order to improve the thermoelectric power factor in multivalley semiconductors and molecular thermoelectrics from a Landauer perspective. A band structure possessing a heavy $m^{*}$ upper band with a minimum of about $5 k_{B} T$ above the lower, dispersive band is suggested to achieve nonmonotonic Seebeck coefficient, and therefore a large power factor. Alloying with the proper composition, introduction of resonant states, and proper strain engineering were proposed as means to achieve an appropriate band structure.

It should be noted that the above general guidelines for ideal band structure and material properties apply only to homogeneous single-phase semiconductors. As we will see later, in the case of composite semiconductors or metamaterials (e.g., those with embedded nanoscale 
inclusions or solid-state thermionic devices), it is possible to suppress or even overcome many of the trade-offs discussed above.

Finally, good thermoelectrics possess very low values of lattice thermal conductivity, approaching those of amorphous materials. Materials with large atomic weight of the constituent elements and/or complex crystal structures with many atoms per unit cell exhibit such thermoelectrically favorable thermal conductivity values. Readers are encouraged to consult the references $[89,90]$ for detailed discussions of parameters affecting the thermal conductivity of materials.

The materials desirable characteristics discussed above can be summarized in materials indicators, other than weighted mobility, which have been used as screening parameters for materials selection. One of these parameters is a dimensionless parameter called the $B$ factor which was first introduced by Chasmar and Stratton (as $\beta$ coefficient) [43],

\begin{tabular}{|l|l|l|}
\hline & $B=\frac{T k_{\Xi}^{2}}{e k_{l}} N_{v} \mu_{x} \sqrt{m_{1} m_{2} m_{3}}\left(\frac{k_{E} T}{2 \pi h^{2}}\right)^{3 / 2}$ & (6)
\end{tabular}

The higher the $B$ parameter for a material, the higher the ZT would be. [44] $B$ factors for $\mathrm{Bi}_{2} \mathrm{Te}_{3}$ (at $300 \mathrm{~K}), \mathrm{PbTe}($ at $650 \mathrm{~K})$ and $\mathrm{SiGe}($ at $1100 \mathrm{~K})$ are $0.39,0.28$, and 0.38 , respectively.

Later, Tuomi [91] defined the quantity called the quality factor $Q$, which extracts the properties related to carriers from the $B$ factor [46]

\begin{tabular}{|l|l|l|}
\hline & $Q=N_{v} \mu_{x} \sqrt{m_{1} m_{2} m_{3}}$ & (7) \\
\hline
\end{tabular}

The quality factor has dimensional units of the mobility (i.e. $\mathrm{cm}^{2} / \mathrm{V} . \mathrm{s}$ ) and in essence combines the weighted mobility expression with the multivalley band structure criteria. Increasing the quality factor increases $B$, which as a result enhances $Z T$. Room-temperature quality factors (in $\mathrm{cm}^{2} /$ V.s) for several $n$-type semiconductors calculated by Mahan [46] are 4380 (for Si), 1290 (for $\mathrm{Bi}_{2} \mathrm{Te}_{3}$ ), 335 (for $\mathrm{GaAs}$ ), and 160 (for $\mathrm{PbTe}$ ). It should be noted that these values are calculated for low doping concentrations (based on the reported mobility values used).

In a very good but rarely referenced paper, Min and Rowe incorporated the Slack's electronegativity idea with thermal parameter using a so-called "quantum structural diagram (QSD)" on binary compounds to identify potential crystal structures for TE applications. QSD had been used earlier to predict ferroelectrics, high- $\mathrm{T}_{\mathrm{c}}$ superconductors and stable quasi-crystals.

\section{Thermoelectric Power Factor Enhancement Mechanisms}

In this section we review the mechanisms proposed to alleviate the trade-off between electrical conductivity and Seebeck coefficient as well as the materials engineering approaches used to implement such concepts, which result in an improvement of the thermoelectric power factor. The mechanisms are discussed in three main categories, the approaches to mainly enhance the Seebeck coefficient, the ones targeting electrical conductivity quasi-independently, and finally the ideas to completely decouple $\sigma$ and $\alpha$ and simultaneously improve both.

\subsection{Strategies to enhance Seebeck coefficient}

\subsubsection{Quantum confinement}


The very first idea toward the improvement of Seebeck coefficient was theoretically proposed by Hick and Dresselhaus using quantum confinement concept in low-dimensional structures. The emergence of the field of low-dimensional thermoelectricity started with this concept. The idea can be readily explained using Mott formula, Equation (5). By inserting the differential conductivity, $\sigma(E)$, into the formula and taking the derivative, we obtain

$$
\alpha=\left.\frac{\pi^{2} k_{E}^{2} T}{3 e}\left\{\frac{1}{g(E)} \frac{d g(E)}{d E}+\frac{1}{\mu(E)} \frac{d \mu(E)}{d E}\right\}\right|_{E=E_{F}} .
$$

It is observed that sharp features in the electronic density of states are desired in order to increase $d g(E) / d E$, and therefore to improve the Seebeck coefficient. Hicks and Dresselhaus [1] show that as the system size decreases and approaches nanometer length scales, it is possible to induce dramatic differences in the electronic density-of-states as shown in Figure 8, which allows to vary $\alpha$ quasi-independently. Figure 8 shows the appearance of sharp features in the electronic density-of-states as a function of dimensionality following

$$
g(E)=\frac{N_{v}}{g_{D} a^{3-D}}\left(\frac{2 m_{d}}{h^{2}}\right)^{D / 2}\left(E-E_{0}\right)^{\frac{D}{2}-1}
$$

where $a$ is the physical length scale, $N_{v}$ is the number of equivalent valleys, $D$ is "dimensionality' factor which equals 3,2 and 1 for a bulk material, a quantum well and a nanowire, respectively. $\mathrm{g}_{\mathrm{D}}$ is a constant which equals $2 \pi^{2}$ for $D=3$, and $D \pi$ for $D=2,1$. As it was introduced before, $m_{d}$ is the total density of state effective mass which is the geometrical average of the band effective masses, i.e. $m_{d}=\left(\Pi_{i=1}^{3-D} m_{i}\right)^{1 / D}$. At lower dimensions $m_{d}$ does not include the effective mass in the direction of confinement.
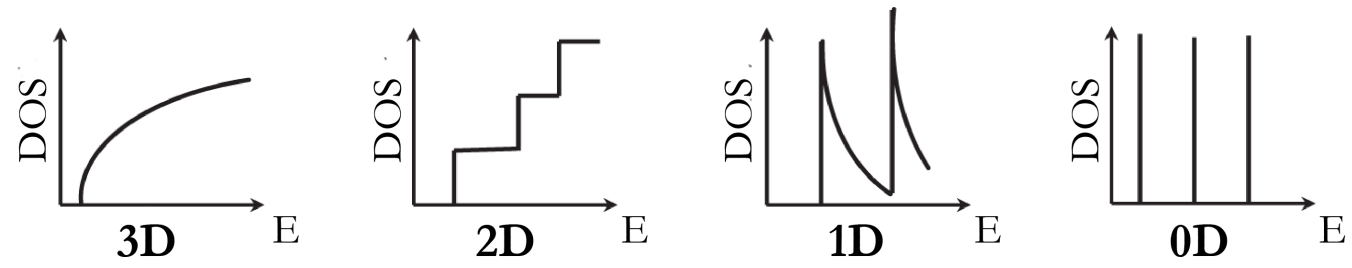

Figure 8 - Evolution of sharp feature in the electronic density of states with dimensionality for a) a bulk 3D crystalline semiconductor, b) a 2D quantum well, c) a 1D nanowire or nanotube, and d) a 0D quantum dot. (Adapted with permission from Ref. [92]. Copyright (C) 2015 by John Wiley \& Sons, Inc.)

Hicks and Dresselhaus [1] then implemented the model on a real material system, $\mathrm{Bi}_{2} \mathrm{Te}_{3}$. The results predicted an increase in $Z T$ by a factor of 13 over the bulk value in the $\mathrm{x}-\mathrm{Z}$ plane for current flow along the $\mathrm{x}$-axis for layers that are $3.8 \AA$ thick. In the case of the $\mathrm{x}-\mathrm{y}$ plane, results still predicted an increase in ZT by a factor of 3 over the bulk value for layers that are $10 \AA$ thick. The dispersion model used in the above work treats the quantum wells as decoupled such that there is no tunneling between the wells. Sofo and Mahan [93] and later Broido and Reinecke [94] showed that additional considerations need to be included in the theory in order to obtain a more realistic understanding of these structures. Broido and Reinecke concluded that a maximum in 
figure of merit of superlattices exist as function of periods of alternating layers. For large periods, they predict a smaller figure of merit than that of the corresponding bulk. [94]

The proof-of-principle demonstration of the concept was first reported for two-dimensional molecular-beam epitaxy (MBE)-grown $n$-type $\mathrm{PbTe} / \mathrm{Pb}_{1-\mathrm{x}} \mathrm{Eu}_{\mathrm{x}} \mathrm{Te}$ quantum well superlattices (QWSL) with 100 to 150 periods and a PbTe quantum well width between 1.7 to $5.5 \mathrm{~nm}$ and a $45 \mathrm{~nm}$ wide $\mathrm{Pb}_{0.927} \mathrm{Eu}_{0.073} \mathrm{Te}$ barrier layer. $[95,96]$ The carrier concentration was tuned by using $\mathrm{Bi}$ as donor atoms in the barrier material. It was experimentally demonstrated that the Seebeck coefficient can be improved relative to the bulk PbTe (by a factor of 1.4 for $5 \mathrm{~nm}$-wide $\mathrm{PbTe}$ QW and 1.8 for $2 \mathrm{~nm}$-wide QW at similar carrier concentration of $8.9 \times 10^{18} \mathrm{~cm}^{-3}$ ) for quantum well widths of $<5 \mathrm{~nm}$ due to the increased density-of-states at each subband edge. They measured MQW mobilities of over $1400 \mathrm{~cm}^{2} / \mathrm{V}$.s, which is comparable to the best PbTe single layer samples (with $1600 \mathrm{~cm}^{2} / \mathrm{V}$.s). Soon after, similar results were reported for $p$-type $\mathrm{PbTe} / \mathrm{PbSe}_{\mathrm{x}} \mathrm{Te}_{1-\mathrm{x}}$ quantum dot superlattices (QDSL). [97,98] The readers are encourages to consult excellent reviews of the superlattice thermoelectrics given by Bottner et al. [99], Venkatasubramanian et al. [100], and Broido and Reinecke [101].

\subsubsection{Electron energy filtering}

The electron energy filtering scheme was earlier proposed for higher power factor in thermionics [102], the other direct thermal-to-electrical energy conversion technology than thermoelectrics. The major difference between thermoelectrics and thermionics is that in a thermionic process the electron transport is ballistic across the energy barrier while in a thermoelectric process the motion of electrons is quasi-equilibrium and diffusive [103]. As was seen in Equation (3), in a quasi-equilibrium and diffusive electron transport process, Seebeck coefficient is, to the first order, proportional to $\left\langle E-E_{F}\right\rangle$. Therefore, at a given carrier concentration, the higher the mean excess energy $\left\langle E-E_{F}\right\rangle$ the higher the $\alpha$. In total, a high $P F$ thus requires a large and energyasymmetric differential conductivity, $\sigma(E)$, within the Fermi window. This is the general rationale behind the electron energy filtering scheme. In light of Equations (2) and (3), the electron energy filtering scheme ensures a higher $\alpha$ via filtering out those electron with lower mean excess energy, but the decreased carrier concentration tends to degrade the $\sigma$. Therefore the energy filtering scheme should be implemented in such a way that the reduction in $\sigma$ is compensated by the increment in $\alpha$ for a higher $P F$. The idea can be implemented by the

introduction of tall barriers $\left(\sim 1-10 \times k_{B} T\right)$ in the conduction band of an $n$-type material or the

valence band of a p-type material. The higher-energy carriers can be selectively transmitted through the structure by filtering out the lower-energy carriers, as shown schematically in Figure 9. This can result in a substantially increased Seebeck coefficient. 


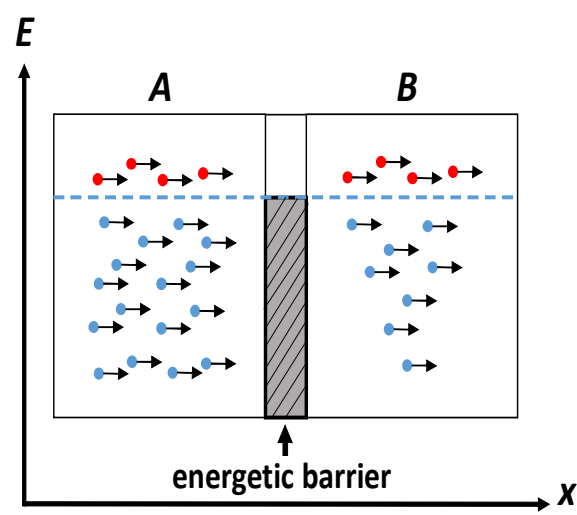

Figure 9 - Schematic diagram of the electron energy filtering mechanism. Among the electrons moving from material "A" to "B", lower energy electrons (blue circles) are more likely to be filtered out by the energetic barrier.

Implementation of small potential barriers that could enhance the power factor was first suggested by Rowe and Min [104] as well as Whitlow and Hirano [105]. Shakouri and Bowers first proposed inserting tall barrier layers into degenerate semiconductor superlattices for selective emission of hot electrons and thus a higher $P F$ [102]. Furthermore, Vashaee and Shakouri pointed out that the lateral momentum must be relaxed to allow a larger number of hot electron emissions in order for a higher PF in the direction perpendicular to planar barriers [106]. As momentum conservation is partially relaxed, energy conservation becomes the major constraint, hence, a key control parameter is the band offset at the barrier-superlattice interface. Evidence for electron energy filtering effect has been found in indium gallium arsenide superlattice films [107]. Kim et al. latterly found that the enhancement in emission current due to the lateral momentum non-conservation is modest because the small number of in-well modes and barrier layers limits the emission current [108]. The technical complications and cost of implementing the planar barrier electron energy filtering scheme in TE materials make people turn to the non-planar barrier electron energy filtering scheme. To this end, a bulk host matrix with discrete nanoparticles embedded [109] is a promising configuration because it requires less precise control of material morphology. At a host-nanoparticle interface, the charge carriers with higher excess energy will pass the interface preferentially, thereby enhancing the thermopower and, in some cases where the carrier mobility does not suffer too much, the $P F$. The high density of interface ensures the strength of filtering effect. For example, in $\mathrm{Pb}$ or $\mathrm{Ag}$ nanoparticles embedded in PbTe bulk host matrix, Nernst coefficient measurements showed that the scattering parameter $r$ changed from $0.2<r<0.7$ to $r>3$ upon introducing nanoinclusions, indicating an electron energy filtering effect, although $r>3$ doesn't correspond to any known scattering mechanism [110]. A larger magnitude of $r$ is thermoelectrically beneficial as long as $\mu$ is not degraded too much. One needs to note that even in the cases with high density of nanoparticles, electron filtering could not be achieved fully in these structures since electron wave could always leak around the nanoparticles. Therefore the case of 1D transport in superlattices is a better geometry for electron filtering if cost was not an issue. To compete with superlattice geometry in a bulk sample, perhaps a better geometry is to coat the nanograins by a filtering layer and then pressing the grains to form a nanocomposite sample. Using a proper coating layer could suppress the grain growth as well as fully confining low energy electrons from all directions. More discussion on the interfacial effect will be given later in the corresponding section. 
There is a long list of experimental reports where energy filtering scheme plays a key role, though not the sole underlying mechanism, in the improved thermoelectric properties of a wide range of materials, especially in nanocomposites and hetero-structures. As a non-exhaustive list, $\mathrm{PbTe}$ nanocomposites [111], solution-processed $\mathrm{Pt}_{-} \mathrm{Sb}_{2} \mathrm{Te}_{3}$ nanocomposites [112], $\mathrm{AgSbTe}_{2}-$ $\mathrm{Ag}_{2} \mathrm{Te}$ nanocomposites [113], $\mathrm{Yb}_{0.26} \mathrm{Co}_{4} \mathrm{Sb}_{12}-\mathrm{GaSb}$ nanocomposites [114], $\mathrm{Bi}_{2} \mathrm{Te}_{2.7} \mathrm{Se}_{0.3}$ nanoplatelet composites [115], organic (P3HT)-inorganic $\left(\mathrm{Bi}_{2} \mathrm{Te}_{3}\right)$ hetero-structures [116], $(\mathrm{Bi}, \mathrm{Sb})_{2} \mathrm{Te}_{3}$-based nanocomposites [117,118], (La,Sr) $\mathrm{TiO}_{3}$ with $\mathrm{Nb}$-doped grain boundaries [119], heavily B-doped Si nanocrystals [120], $\beta-\mathrm{Zn}_{4} \mathrm{Sb}_{3}-(\mathrm{Bi}, \mathrm{Sb})_{2} \mathrm{Te}_{3}$ composites [121], and $\mathrm{Ag} / \mathrm{oxide} / \mathrm{Sb}_{2} \mathrm{Te}_{3}-\mathrm{Te}$ hetero-structures [122]. In particular, electron energy filtering mechanism contributed to the rare simultaneous improvement in all three thermoelectric properties in $p$-type $\mathrm{Ti}(\mathrm{Co}, \mathrm{Fe}) \mathrm{Sb}$-InSb nanocomposites [123]. Theoretically, the electron energy filtering effect has been investigated in semiconductor-metal nanocomposites [124], and $\mathrm{Mg}_{2} \mathrm{Si}_{\mathrm{x}} \mathrm{Sn}_{\mathrm{x}}$ solid solutions [125].

\subsubsection{Resonance levels}

By resonant scattering we mean the scattering parameter $r$ not only strongly depends on energy but also resonates at certain energies [126,127] Resonance levels (RLs) mechanism, occasionally called "virtual bound states" [128], is a unique type of resonant scattering. In thermoelectricity, the role of RLs is hierarchically dual. First, RLs induce narrow peak in the electronic density-ofstates (DOS) that centers at the resonant energy $E_{D}$ and often adopts a Lorentzian line shape. Second, RLs conduct current while resonantly scatter conduction electrons. The conductionwhile-resonantly scattering role makes RLs unique. In quantum mechanical partial-wave analysis, the resonant scattering mechanism of RLs is properly described in terms of the phase angle shift $\delta_{l}$ of $l$ th partial wave caused by a potential, where $l$ is the orbital angular momentum quantum number [129]. Unlike other scattering mechanisms simply labeled as momentum relaxation or energy relaxation, the RLs mechanism is, properly speaking, a "dephasing process", as is shown schematically in Figure 10. 


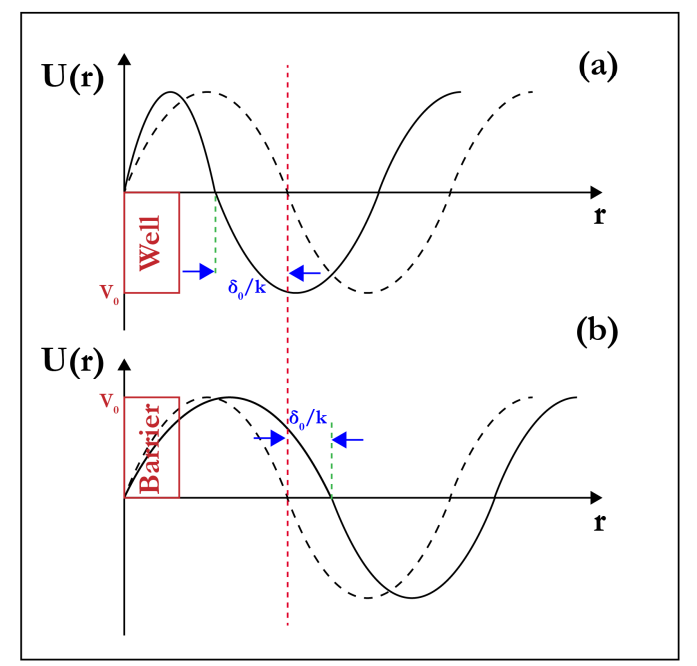

Figure 10 - A schematic visualization of the phase shift $\delta_{0}$ caused by (a) a spherical finite square well $\left(\delta_{0}>0\right)$ and (b) a spherical finite square barrier $\left(\delta_{0}<0\right)$. The dashed line and the solid line represent the radial wave function before and after phase shift, respectively. The parameters $V_{0}$ and $k$ are the potential depth/height and wave number of the matter wave, respectively.

The detailed energy dependence of phase angle shift further defines two important parameters. First, the rise of $\delta_{l}$ with increasing energy $E$ is physically associated with "a delay of the emergence of the trapped particle" in the context of time-dependent Schrödinger equation [129], i.e., Wigner delay time $\tau_{\mathrm{w}}=2 \hbar \frac{\partial \delta_{2}(\boldsymbol{E})}{\partial E}$ [130]. In practice, $\tau_{\mathrm{w}}$ can be regarded as the amount of time that a conduction electron is momentarily "trapped" at an impurity atom before resuming its conduction. Second, we define a characteristic energy interval $\Gamma$ over which $\delta_{l}$ changes from 0 to $\pi$. In the simple case of $s$ - $d$ level hybridization [131], $\Gamma=2 \pi|\zeta l| V|k>|^{2} g(E)$, where V is the effective $s$ - $d$ interaction term, $g(E)$ the electron density of states at energy $E$ for those extended eigenstates, $|l\rangle$ and $\mid k>$ respectively the localized and extended eigenstates. Qualitatively, $\Gamma$ is inversely correlated with $\tau_{\mathrm{w}}$. Resonant scattering for the $l$ th partial wave literally occurs at $\delta_{l}=$ $\pi / 2$, where the $l$ th partial wave scattering cross-section reaches the maximum at the resonance energy $E_{D}$. In the vicinity of $E_{D}, \tau_{\mathrm{w}}$ usually exhibits a sharp peak, indicating conduction electrons are scattered in a way that is sensitive to their energy. $E_{D}$ and $\Gamma$ are the two major control parameters of RLs in TE research. The main theme of this section is thus how to optimize these two parameters to enhance the $P F$ via proper material implementations. Readers are referred to reviews by Ravich [127] and Heremans [6,132] for more technical details of the RLs mechanism.

As it was mentioned above, RLs play a dual role in thermoelectricity. The first major impact of RLs on the $P F$ comes from "resonant scattering". In general, strong energy-dependent scattering of charge carriers is thermoelectrically favorable [47]. To implement RLs in TE materials, most of which are narrow band gap semiconductors, the position of $E_{D}$ relative to the band edge and the position of $E_{D}$ relative to the chemical potential, $\mathrm{E}_{\mathrm{F}}$, are equally important for the RLs mechanism to function as desired. RLs are usually implemented via hetero-electron doping. Notably, resonant dopant impurities must have electron energy levels $E_{\mathrm{D}}$ falling in the conduction band or the valence band, rather than falling inside the band gap. 
The other major impact comes from the RLs-induced distortion of electron density-of-states (DOS). For the purpose of illustration, we schematically plot a RLs-distorted conduction band DOS in Figure 11. RLs induce an excess DOS peak centered near $E_{D}$ and with the width $\Gamma$. The excess DOS peak is on top of the so-called background DOS, which arises from the band(s) without RLs. After Mahan and Sofo [79], the lower the background DOS, the higher the excess DOS peak, the narrower the $\Gamma$, the higher the $P F$ will be, given an optimally positioned chemical potential, $E_{F}$. In practice, one needs a $\Gamma \sim 10-100 \mathrm{meV}$ to allow $E_{F}$ inside the RLs; the background DOS itself is detrimental for high $P F$ but it is necessary for precise positioning of $E_{F}$; the RLs must conduct heat and charge (thus imposing an upper requirement on $\tau_{\mathrm{w}}$ ), otherwise the RLs basically leads to less thermoelectrically favorable chemical potential pinning [133]. One should also note that allowing $E_{F}$ and $E_{D}$ within a narrow DOS peak will risk some free energy instability, for this reason, $E_{F}$ tends to stay near a local minima, not a local maxima [134].

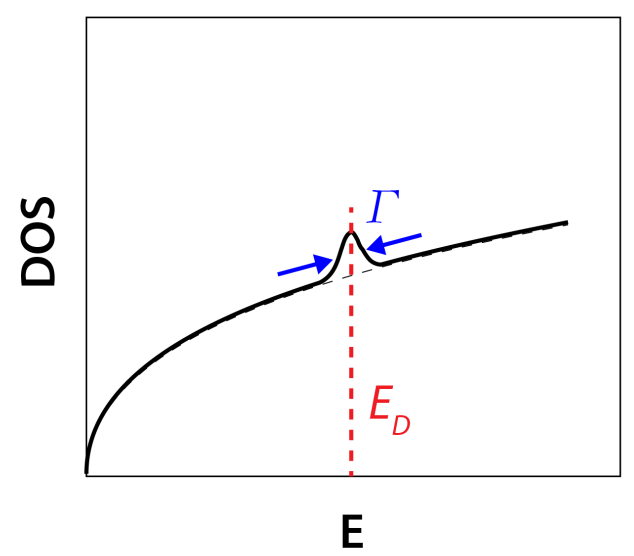

Figure 11 - Schematic electron density of states (DOS). $E_{D}$ is the resonance level and $\Gamma$ the resonance width.

Owing to the inter-dependence of the electrical conductivity $(\sigma)$ and the electronic thermal conductivity $\left(\kappa_{\mathrm{e}}\right)$ in the definition of $Z T$, it is desirable that the enhancement in the $P F$ comes mainly from the enhancement in the thermopower $(\alpha) .{ }^{3}$ In order to discuss the impact of RLs on $\alpha$, we can refer to Equation (8). It is instructive to note that the two terms in the braces of Equation (8) each corresponds to a mechanism that affects $\alpha$. What is unique about RLs is that they simultaneously affect both mechanisms.

In Equation (8), $g(E)$ is the result from the hybridization between the localized atomic levels of impurity atom and the extended eigenstates of conduction electron that are close in energy, i.e., $g(E)$ is the RLs-induced DOS peak plus the background DOS. The impact of DOS peak on the $P F$ and $Z T$ of bulk TE materials was discussed earlier. To cover the emerging nanostructured TE materials, Pichanusakorn and Bandaru [4] adopted a semi-classical Boltzmann transport model and reached several enlightening conclusions: (i) "the sheer magnitude and not the specific shape" of the electron density of states (DOS) is the key to enhancing the PF; (ii) assuming parabolic band and power-law scattering mechanism, the $P F$ will be maximized when $\alpha$ is in the range of $\sim 130-187 \mu \mathrm{V} / \mathrm{K}$, consistent with experimental observation in a wide variety of TE

\footnotetext{
${ }^{3}$ For the purpose of illustration, the thermopower has to be greater than $225(275) \mu \mathrm{V} / \mathrm{K}$ to ensure a ZT $\sim 2.0(3.0)$ for a hypothetical material in which $\kappa_{\mathrm{ph}}$ is zero and the Wiedemann-Franz relationship holds. A ZT $\sim 3$ material will be a game changer in the areas of waste heat recovery and heat management.
} 
materials; (iii) the $P F$ is inherently limited by the onset of the bipolar conduction at elevated temperatures; and ( $i v)$ the $P F$ of a nanomaterial can be enhanced beyond its bulk counterpart via quantum confinement induced DOS enhancement at $\mathrm{E}_{\mathrm{F}}$.

One should note that there are several inherent limitations on the implementations of RLs mechanism in TE materials. First, the RLs-induced DOS peak must actively contribute to charge conduction. This argument is supported by the contrasting results in Tl-doped PbTe [135] and Ti-doped PbTe [136]. Second, $\Gamma\left(\tau_{\mathrm{w}}\right)$ cannot be too narrow (too long) in light of the formula $\Gamma=$ $2 \pi|<l| V|k>|^{2} g(s)$. As such, $3 d$-state and $f$-state are less practical for RLs because of their narrowness and also the difficulty of properly positioning $\mathrm{E}_{\mathrm{F}}$ in practice. So the resonant impurity states should be of $s$ - or $p$-like character. It is intuitive to note that although any $s$-state by itself lacks peak feature in its specific DOS the Tl $6 s$-state induces sharp DOS peak near the band edge (where the background DOS is minimal) in PbTe upon hybridization with Te $5 p$ state [6]. In this specific case, Tl $6 s$-state is resonant yet nearly free. On the other hand, $\Gamma\left(\tau_{\mathrm{w}}\right)$ can't be too broad (too short), otherwise the effect of RLs will be weak. Thus far, the most viable optimization route is through the proper selection of the host matrix governing the background DOS and the $\mid l>$ states that contribute to resonance. In summary, successful implementation of RLs is a delicate trade-off.

In the context of relaxation time approximation, the relaxation time for RLs-induced resonant scattering $\tau_{\mathrm{r}}$ can be expressed as [127],

$$
\tau_{r}(E)=\tau_{0}\left[1+\left(\frac{E-E_{D}}{\Gamma / 2}\right)^{2}\right]
$$

where $\tau_{0}$ is the minimum value of $\tau_{r}(E)$ reached at $E_{D}$. Equation (10) can be incorporated into Matthiessen's rule $\left(1 / \tau=\Sigma_{i} 1 / \tau_{i}\right.$, where $\tau_{i}$ is the relaxation time for the $i^{\text {th }}$ scattering channels, $\tau$ the total relaxation time) for further data analysis. When the RLs mechanism is the dominant scattering mechanism, the strong energy dependence of $\tau_{\mathrm{r}}$ (Equation (10)) renders a strongly energy-dependent $\tau$ and thus a strongly energy-dependent $\mu(E)$ (from $\mu(E)=e \tau(E) / \mathrm{m}^{*}$ ). Therefore, the term $1 / \mu(E)(d \mu(E) / d E)$ in Equation (8) dominates. It is worth noting that the impact of RLs on the mechanism is $(i)$ weakly temperature-dependent because the electron band structure is, to the first order, temperature independent and the only temperature dependent factor is from the Fermi-Dirac distribution function, and (ii) more pronounced at lower temperature (cryogenic temperatures) as the acoustic phonon scattering dominates at higher temperatures.

Calculated partial DOS of $\mathrm{Tl}$ atom near $\mathrm{E}_{\mathrm{F}}$ in $\mathrm{Pb}_{0.98} \mathrm{Tl}_{0.02} \mathrm{Te}_{1-\mathrm{x}} \mathrm{S}_{\mathrm{x}}$ and the corresponding resonant "hump" is shown in Figure 12.[135] There are a number of confirmed examples of RLs in TE materials, some are successful in enhancing the $P F$ [37,137], some less so but still illustrative $[136,138,139,140,141]$. In most cases, a deviation from the Pisarenko-like behavior, especially a thermopower significantly higher than what a Pisarenko curve predicts, suggests the presence of the RLs mechanism as shown in Figure 13 [132]. Certainly, the deviation by itself cannot pinpoint the RLs. 


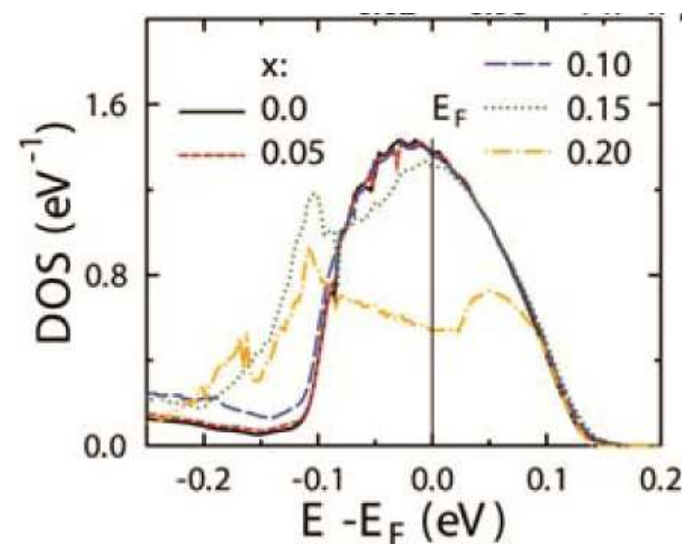

Figure 12 - Partial DOS of $\mathrm{Tl}$ atom (mostly s-like) near $\mathrm{E}_{\mathrm{F}}$ in $\mathrm{Pb}_{0.98} \mathrm{Tl}_{0.02} \mathrm{Te}_{1-\mathrm{x}} \mathrm{S}_{\mathrm{x}}$ and its evolution as a function of $\mathrm{S}$ content. Up to $\mathrm{x}=0.1$, the resonant "hump" is not $\mathrm{x}$-dependent; for $\mathrm{x} \geq 15 \%$ it is lowered, broadened, and starts to join the main valence DOS. (Reproduced from Ref. [135] with permission from The Royal Society of Chemistry)

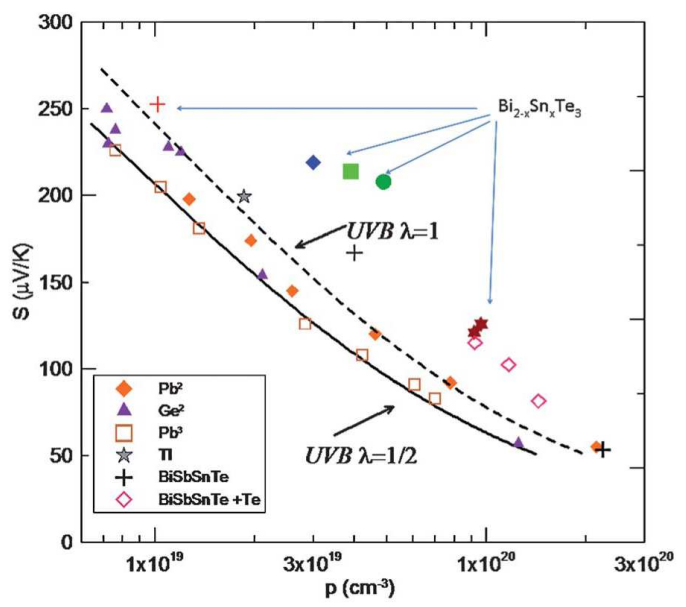

Figure 13 - Room temperature Pisarenko plot for $p$-type $\mathrm{Bi}_{2} \mathrm{Te}_{3}$. The lines are calculated results, while the data points are obtained for the different acceptor impurities as marked, by different groups. The points where Sn is used as a dopant differ significantly from the calculated lines, a signature of RLs. The calculation assumed a density-of-states effective mass of $0.35 m_{e}$. (Reproduced from Ref. [6] with permission from The Royal Society of Chemistry)

Above we have shown that the enhancement in the $P F$ and the $\alpha$ can be attained via electron energy filtering, either non-resonant or resonant. It is thus important to experimentally differentiate the specific contribution from each mechanism, especially in light of the fact that the theoretical modeling by itself cannot predict all the details of $\mathrm{RL}_{\mathrm{S}}$ and its impact on the TE properties. To this end, thermo-galvano-magnetic effects are evoked especially the low magnetic field Nernst effect and high magnetic field thermopower. Particularly, if only one type of carrier is present, one can apply the so-called "method of the four-coefficients" to determine the carrier concentration, mobility, scattering parameter/exponent, and effective mass from the results of the electrical conductivity, thermopower, Hall coefficient, and Nernst coefficient [110]. 


\subsection{Strategies to enhance electrical conductivity}

\subsubsection{Modulation doping}

It was pointed out that most of the thermoelectric materials are heavily doped semiconductors with carrier concentration on the order of $10^{18} \mathrm{~cm}^{-3}$ to $10^{20} \mathrm{~cm}^{-3}$. The high level of the doping is required to provide the high density of charge carriers required to conduct electricity. At the same time, high carrier mobility is required to transfer electricity in an efficient manner. Addition of doping centers, provide the required level of conduction carriers. However, the ionized impurity centers left behind in the lattice, serve as scattering centers to disturb conduction carriers.

The problem of ionized impurity scattering has been addressed long time ago in the context of 2D structures by spatially separating the conduction carriers from their parent atoms. The technique is called modulation-doping, remote-doping or delta-doping. In this scheme, all dopants are concentrated in a thin doping layer, which is separated from the main transport channel by a spacer. The band alignment between the doped region, the spacer and the channel favors carrier transport from the doped region to the channel to spatially separate carriers from their parent atoms and therefore to reduce the ionized impurity scattering. 2D modulation-doping has advanced the semiconductor industry in the past significantly; new efficient transistors (HEMT/HFET/ MODFETs) and optoelectronic devices have been designed. An amazing four orders of magnitude enhancement in the mobility has been achieved over 22 years. The first demonstration of mobility enhancement was in 1978, which demonstrated a mobility of $10^{4}$ $\mathrm{cm}^{2} /$ V.s compared to the bulk mobility of $3 \times 10^{3} \mathrm{~cm}^{2} /$ V.s. In 2000 , the mobility values achieved 31 million $\mathrm{cm}^{2} /$ Vs (see Figure 14). This was mostly the result of advances in MBE technique and a better understanding of the limiting factors. 


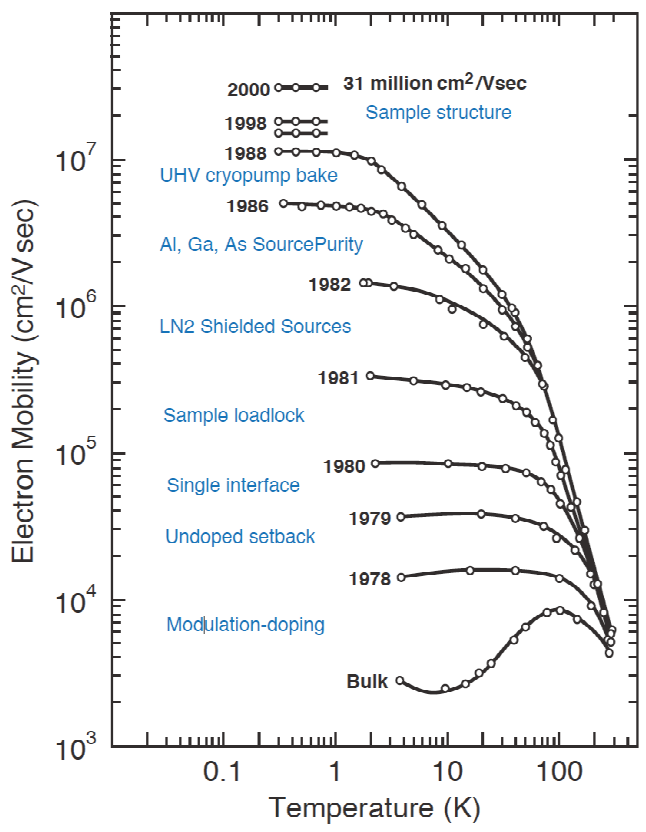

Figure 14 - Historical graph indicating the advances in the mobility enhancement using 2D modulation doping in AlGaAs/GaAs interface (Reprinted from Ref. [142], Copyright (C) 2015, with permission from Elsevier)

In the geometry used in 2D modulation doping, charge carriers are trapped at the interface of the channel and the spacer and move along the interface. Therefore a smooth and clean interface is crucial to minimize roughness scattering, which is only possible by using MBE technique.

Thermoelectric materials used in commercial applications are bulk semiconductors and they have to be cost effective in order to find a possible market. In addition, thermoelectrics are mostly working at high temperatures wherein reasonable ZT values are achievable. As can be seen in Figure 14, modulation doping is mostly significant at low temperatures where ionized impurity scattering is the dominant scattering. At higher temperatures phonon scattering dominates and the enhancement as a result of remote-doping is minor. Therefore, it is challenging to apply the same strategy to this class of materials.

There are few possibilities to apply modulation-doping concept to bulk samples. One possibility is to embed metallic or semi-metallic nanoparticles inside an intrinsic bulk sample. Several groups have demonstrated this so far. G. Chen and Z. Ren groups were the first to demonstrate the possibility of observing improved carrier mobility in nanostructured SiGe samples. [22,143] They have shown that by embedding heavily-doped silicon nanograins (20-50nm in size) inside $\mathrm{SiGe}$ host matrix, it is possible to enhance the thermoelectric power factor by about $40 \%$ compared to conventional SiGe uniform nanostructures doped with boron. In this geometry, uniform doping is replaced by cluster doping. Charge carriers spill over from the clusters to the host matrix and travel within the host matrix with reduced ionized impurity scattering. However, the coulomb interaction between the clusters and the charge carriers is still comparable with the coulomb interaction between single impurities and charge carriers and the charge carriers are not completely separated from their parent atoms due to lack of a space layer. Recently, Wu et al. [144] reported significant enhancement in the power factor and the figure of merit of modulation-doped BiAgSeS in BiAgSeS/BiAgSeS ${ }_{1-\mathrm{x}} \mathrm{Cl}_{\mathrm{x}}$ composites. 
Adding several nanometers of a coating layer to the nanograins could further separate charge carriers and parent atoms and result in further improvement in the carrier mobility. To our knowledge there has not been any demonstration of the effect of the spacer layer in 3D modulation doping. In the case of $2 \mathrm{D} \mathrm{MnSi} \mathrm{i}_{1.7}$ films, Huo et al. looked at the effect of modulation doping for thermoelectric application. In this structure, a heavily doped silicon layer (doped by $\mathrm{Al}$ and $\mathrm{Cu}$ ) is separated from a $\mathrm{MnSi}_{1.7}$ film by a thin layer of silicon $(<20 \mathrm{~nm})$, which serves as the spacer layer. Consequently, Hou et al. were able to improve the power factor of $\mathrm{MnSi}_{1.7}$ films by a factor of two. [145,146]

Another way of remote doping is field-effect doping, wherein carriers are induced inside the transport channel by means of gating. This is important especially for device design as well as the proof of concept of the effectiveness of remote doping to enhance the TE power factor. Liang et al. demonstrated the possibility of tuning Seebeck coefficient of single PbSe nanowires using field-effect gated device. [147] Curtin et al. demonstrated this concept in silicon nanowires. They have shown that gated silicon nanowires could have slightly larger thermoelectric power factors as opposed to bulk silicon conventionally $n$-doped silicon. [148]

The field of thermoelectric modulation-doping is new and is not well investigated yet. The role of the spacer layer is not investigated experimentally due to challenges of making core-shell nanoparticles or nanorods. Other possible geometries such as aligned nanowires inside a host matrix or holey host matrices with filled dopants are yet to be explored.

\subsubsection{Crystallite alignment}

Highly anisotropic nature of many thermoelectric materials, provide good opportunity to improve the properties of corresponding polycrystalline samples by preferentially aligning the crystallites (grains) along the favorable transport directions (typically the high-mobility direction). The goal is to regain the lost properties because of the random orientation of the grains in the polycrystalline sample. Anisotropy ratio of the transport properties of some highly anisotropic single crystalline thermoelctrics are listed in Table 1.

Table 1 - Anisotropy ratio of transport properties in several single crystalline thermoelectric materials at room temperature. Subscripts $a b$ and $c$ refers to in-plane and out-of-plane properties, respectively. In case of the bismuth telluride alloys family, they correspond to 11 (parallel to the cleavage planes) and 33 (perpendicular to the cleavage planes) directions typically used in thermoelectrics literature.

\begin{tabular}{|cccccc|}
\hline Material & $n$ or $p\left(\mathrm{~cm}^{-3}\right)$ & $\alpha_{\mathrm{ab} /} \alpha_{\mathrm{c}}$ & $\rho_{\mathrm{cl}} \rho_{\mathrm{ab}}$ & $\kappa_{\mathrm{ab} /} \kappa_{\mathrm{c}}$ & Reference \\
\hline $\mathbf{B i}_{\mathbf{0 . 5}} \mathbf{S b}_{\mathbf{1 . 5}} \mathbf{T e}_{\mathbf{3}}$ & $3.3 \times 10^{19}$ & $\sim 1$ & 3 & 1.8 & {$[149]$} \\
\hline $\mathbf{B i}_{\mathbf{0 . 4}} \mathbf{S b}_{\mathbf{1 . 6}} \mathbf{T e}_{\mathbf{3}}$ & $5.9 \times 10^{19}$ & 0.88 & 2.6 & 2.15 & {$[149]$} \\
\hline $\mathbf{B i}_{\mathbf{2}} \mathbf{T e}_{\mathbf{2 . 8 5}} \mathbf{S e}_{\mathbf{0 . 1 5}}$ & $4 \times 10^{19}$ & 1.04 & 5.05 & 2.21 & {$[150]$} \\
\hline $\mathbf{C a}_{\mathbf{3}} \mathbf{C o}_{\mathbf{4}} \mathbf{O}_{\mathbf{9}}$ & - & $1-2$ & $5-15.5$ & $2-3$ & {$[183]$} \\
\hline $\mathbf{N a C o}_{\mathbf{2}} \mathbf{O}_{\mathbf{4}}$ & $2.4 \times 10^{21}$ & - & 42 & - & {$[153]$} \\
\hline $\mathbf{M n S i} \mathbf{i}_{\mathbf{1 . 7 3}}$ & $\sim 10^{21}$ & $1 / 2.1$ & 8.5 & 1.5 & {$[151,152]$} \\
\hline
\end{tabular}


One of the most-studied material systems for control of the crystallographic texture is $p$-type $\left(\mathrm{Bi}_{\mathrm{x}} \mathrm{Sb}_{1-\mathrm{x}}\right)_{2} \mathrm{Te}_{3}$. As it is listed in Table 1, different solid solutions of $\mathrm{Bi}_{2} \mathrm{Te}_{3}-\mathrm{Sb}_{2} \mathrm{Te}_{3}$ exhibit different degrees of anisotropy. Caillat et al. [149] systematically investigated the anisotropic electrical and thermal transport in $\left(\mathrm{Bi}_{\mathrm{x}} \mathrm{Sb}_{1-\mathrm{x}}\right)_{2} \mathrm{Te}_{3}$ single crystal solid solutions. It was found that even though the electronic transport shows strong anisotropy in the in-plane versus out-of-plane directions, $\mathrm{ZT}$ is nearly isotropic $\left(\mathrm{Z}_{11} \approx \mathrm{Z}_{33}\right)$ for $\mathrm{Bi}_{0.4} \mathrm{Sb}_{1.6} \mathrm{Te}_{3}$ single crystals (at $300 \mathrm{~K}$ ), for all carrier concentrations studied. However, $\mathrm{Bi}_{0.5} \mathrm{Sb}_{1.5} \mathrm{Te}_{3}$ single crystals exhibit an anisotropy ratio in the figure of merit of $\mathrm{ZT}_{11} / \mathrm{ZT}_{33}=1.73$ originating from an almost isotropic Seebeck coefficient along with a strong anisotropy in electrical conductivity. This suggests $\mathrm{Bi}_{0.5} \mathrm{Sb}_{1.5} \mathrm{Te}_{3}$ to be the ideal candidates among the solid solutions studied for crystallite alignment in the polycrystalline samples. It is worth mentioning that Caillat et al.[149] reported the highest figure of merit for $\mathrm{Bi}_{0.45} \mathrm{Sb}_{1.55} \mathrm{Te}_{3}$ solid solutions for which the in-plane transport properties are only reported. A strong anisotropy was also reported for $n$-type $\mathrm{Bi}_{2}\left(\mathrm{Te}_{1-\mathrm{x}} \mathrm{Se}_{\mathrm{x}}\right)_{3}$ single crystals. Carle et al.[150] reported an almost isotropic Seebeck coefficient in $\mathrm{Bi}_{2} \mathrm{Te}_{2.85} \mathrm{Se}_{0.15}$ while possessing a strong anisotropy in electrical conductivity which leads to a larger power factor (a factor of 5) in the out-of-plane direction. An anisotropy in the figure of merit of $\mathrm{ZT}_{11} / \mathrm{ZT}_{33}>2.5$ is observed in $\mathrm{Bi}_{2}\left(\mathrm{Te}_{1-\mathrm{x}} \mathrm{Se}_{\mathrm{x}}\right)_{3}$ single crystal solid solutions.

The chimney-ladder structured higher manganese silicide (HMS) single crystal is also a good example of yet another thermoelectric compound with highly anisotropic transport properties over a wider temperature range. Large anisotropy in the electrical conductivity leads to a larger power factor (a factor of 2 at $300 \mathrm{~K}$ ) and figure of merit in the a-b plane (perpendicular to the caxis).

Another class of highly anisotropic thermoelectric materials is layered oxide thermoelectrics, among which sodium $\left(\mathrm{Na}_{\mathrm{x}} \mathrm{CoO}_{2}\right)$ and calcium $\left(\mathrm{Ca}_{3} \mathrm{Co}_{4} \mathrm{O}_{9}\right)$ cobaltite have attracted much attention. In cobaltite, due to the insulating $\mathrm{CoO}$ layers, the electrical conductivity is higher in the $a-b$ plane than along the c-axis. Terasaki et al. [153] reported the first results on the anisotropic electronic transport in the $\mathrm{Na}_{\mathrm{x}} \mathrm{CoO}_{2}$ single crystal, which triggered the exploration of oxide thermoelectric materials. They reported a very strong anisotropy in the in-plane electrical conductivity (by a factor of 42 , at $300 \mathrm{~K}$ ) leading to a large in-plane power factor of $1.5 \mathrm{Wm}^{-1} \mathrm{~K}^{-}$ 1 , larger than that of the bismuth telluride $\left(1.2 \mathrm{Wm}^{-1} \mathrm{~K}^{-1}\right)$ at room temperature. Later Sales et al. [154] reported a colossal anisotropy ratio in the in-plane vs. out-of-plane electrical conductivity of $\sim 550$ for $\mathrm{Na}_{0.75} \mathrm{CoO}_{2}$. To the best of our knowledge, there are no reports on the out-of-plane thermopower of $\mathrm{Na}_{\mathrm{x}} \mathrm{CoO}_{2}$ single crystals. In the case of misfit-layered $\mathrm{Ca}_{3} \mathrm{Co}_{4} \mathrm{O}_{9}$ single crystals, different anisotropy ratio of electrical transport is reported for crystals prepared by different groups. Masset et al.[183] reported an anisotropy in the electrical resistivity of $\rho_{\mathrm{c} /} \rho_{\mathrm{a}}=15.5$ at room temperature whereas Bhattacharya et al. [155] reported a ratio of 4.8. The anisotropic behavior of Seebeck coefficient is less studied in these compounds. Tang et al. [156] reported a factor of 2 variations in the in-plane vs. out-of-plane thermopower in calcium cobaltite.

Several approaches were proposed and successfully implemented to date to take advantage of the anisotropy discussed above in order to artificially align the grains of polycrystalline samples along favorable transport directions. Mechanical alignment of the grains under uniaxial compression is one of the simplest approaches to induce preferential orientation in a polycrystalline sample. This is typically achieved during the consolidation of the power using hot 
press or spark plasma sintering techniques. The degree of alignment depends upon several parameters such as the mechanical properties of the powder, the powder morphology and size distribution, as well as the compressional load applied. The degree of alignment (texture) can be quantified using the orientation factor (also known as Lotgering factor), $F$, by comparing the $\mathrm{X}$ ray diffraction patterns of the aligned specimen with those of the randomly-oriented samples following Lotgering's method. [157] The orientation factor is then defined as

$$
F=\frac{p-p_{0}}{1-p_{0}}
$$

where

$$
p=\frac{\sum I(00 l)}{\sum I(h k l)}
$$

and $p=p_{0}$ for randomly-oriented specimen. $F$ varies from 0 (for randomly-oriented) to 1 (for completely oriented).

Ben-Yehuda et al. reported c-axis oriented polycrystalline $\mathrm{Bi}_{0.4} \mathrm{Sb}_{1.6} \mathrm{Te}_{3}$ prepared from pulverized ingot (average grain size 100-175 micron) possessing $~ 2$ times larger power factor perpendicular to the c-axis which originates from a similar anisotropy in the electrical conductivity.[158,159] Texturing was induced using high pressure $(800 \mathrm{MPa})$ cold compaction and pressure less sintering. The c-axis aligned sample exhibits maximum ZT value of 1 , similar to that of the corresponding single crystal. However, the degree of anisotropy reported for different transport properties is different than what is expected for $\mathrm{Bi}_{0.4} \mathrm{Sb}_{1.6} \mathrm{Te}_{3}$ single crystals by Caillat et al. and is very similar to $\mathrm{Bi}_{0.5} \mathrm{Sb}_{1.5} \mathrm{Te}_{3}$. Due to the composition-dependent nature of the anisotropy in $\mathrm{Bi}$ $\mathrm{Sb}$-Te solid solutions, careful investigation of the chemical composition is crucial in understanding the grain alignment related improvements. Shen et al. [160] also investigated the preferred orientation in large grain $p$-type $\mathrm{Bi}_{0.4} \mathrm{Sb}_{1.6} \mathrm{Te}_{3}$ and n-type $\mathrm{Bi}_{2} \mathrm{Te}_{2.1} \mathrm{Se}_{0.9}$ polycrystalline sample using uniaxial compression (50 $\mathrm{MPa}$ ) with spark plasma sintering technique. A strong anisotropy in electrical conductivity of both samples lead to a larger power factor (30\% larger perpendicular to c-axis). However, due to the strong anisotropy in thermal conductivity, the overall ZT was isotropic, in agreement with the properties of $\mathrm{Bi}_{0.4} \mathrm{Sb}_{1.6} \mathrm{Te}_{3}$ single crystals. Other groups report similar results for large grain (> 50 micron) powders. However, due to the large gain size, higher figure of merit than that of the bulk nanostructured sample has not been reported. The degree of re-orientation achieved using uniaxial compression alone is typically much smaller than the other methods discussed in this section.

Methods of severe plastic deformation such as Hot Area Reduction Extrusion (HARE, which is commonly known as hot-extrusion), Equal Channel Angular Extrusion/Pressing (ECAE or ECAP), and hot-forging are another approach used to produce strongly textured materials. Very high degree of alignment can be achieved using these techniques. However, many of these methods to some extent require ductility. Hot extrusion-based methods comprise the first attempts to improve the thermoelectric properties of thermoelectric materials, particularly those of $\mathrm{Bi}_{2} \mathrm{Te}_{3}$-based alloys [161,162,163] by crystallite alignment (and grain refinement). Hot extrusion-based processes involve the squeeze of a heated billet (cold or hot pressed) through a die.[164] If the heated billet passes through a unidirectional channel and is reduced in diameter, it is referred to as Hot Area Reduction Extrusion (HARE),[165] and if it is forced through a 
channel of constant diameter containing a bend, it is known as Equal Channel Angular Extrusion/Pressing (ECAE or ECAP)[166,167]. Kim et al. [168] reported a high thermoelectric power factor of $1.3 \mathrm{Wm}^{-1} \mathrm{~K}^{-1}$ at room temperature for the shear-extruded $\mathrm{Bi}_{0.4} \mathrm{Sb}_{1.6} \mathrm{Te}_{3}$. The shearextrusion process provides twice higher orientation factors $(\mathrm{F}=0.63)$ than attained by the conventional process, for any $\mathrm{Bi}-\mathrm{Te}-\mathrm{Sb}$ solid solution. This led to a significant increase in the electrical conductivity by $\sim$ a factor of 2 , comparing to the corresponding hot-pressed sample. Similar results were reported by Hayashi et al. [169] for angular-extruded $\mathrm{Bi}_{0.4} \mathrm{Sb}_{1.6} \mathrm{Te}_{3}$. A thermoelectric power factor value of $1.44 \mathrm{Wm}^{-1} \mathrm{~K}^{-1}$, similar to that of the corresponding single crystal, and a ZT $\sim 1$ was achieved mainly due to the enhancement of electrical conductivity and carrier mobility parallel to the extrusion direction. However, these results have been reported for average grain size on the order of $>1$ micron which can be further improved if combined with nanostructuring. Dehkordi and Vashaee[170] reported a 50\% improvement in the power factor of the highly-textured $(\mathrm{F} \sim 0.4)$ p-type $\mathrm{Bi}_{0.5} \mathrm{Sb}_{1.5} \mathrm{Te}_{3}$ nanostructured bulk over that of the randomly-oriented hot pressed sample, using an extrusion-like modified hot-press technique. Larger thermoelectric power factor (above $1.5 \mathrm{Wm}^{-1} \mathrm{~K}^{-1}$ ) than the corresponding single crystal has been reported at $330 \mathrm{~K}$ for the c-axis oriented sample parallel to the extrusion direction. Due to the ductility needed in the hot-extrusion-based alignment, these techniques are most widely investigated for $\mathrm{Bi}_{2} \mathrm{Te}_{3}$-based alloys and not in other anisotropic thermoelectric compounds such as oxides.

In recent years, much attention has been given to texture engineering of thermoelectric materials using yet another method of plastic deformation, namely hot forging (sometimes referred to as "re-pressing" in thermoelectric literature). In hot forging, the sintered bulk sample is placed in a die or mold with larger dimensions than its size and is deformed under a uniaxial compressional load to form the shape of the die in which it was placed. This would generally induce a preferential orientation perpendicular to the forging direction. Shen et al. [171] reported a maximum ZT of 1.1 at room temperature for the hot-forged polycrystalline $p$-type $\mathrm{Bi}_{0.5} \mathrm{Sb}_{1.5} \mathrm{Te}_{3}$. The enhanced figure of merit is, however, a result of a much reduced thermal conductivity. The power factor is slightly reduced. An unexpected increase in the magnitude and temperature dependence of Seebeck coefficient is also observed for all the hot-forged samples comparing to the corresponding single-hot-pressed. Recently, Zhu et al. [172] reported a ZT 1.3 near room temperature for hot-forged $\mathrm{Bi}_{0.5} \mathrm{Sb}_{1.5} \mathrm{Te}_{3}$ zone-melted commercial ingot (50\% improvement over the non-deformed ingot). Ingots hot-forged along different directions exhibit relatively isotropic $\sigma$ and $\kappa$. However, a large anisotropy in-plane vs. cross-plane thermopower is observed, which increases with temperature and reaches a maximum at $500 \mathrm{~K}\left(\alpha_{11 /} \alpha_{33}=1.5\right)$. Temperaturedependence of the thermopower is also observed to be different along different directions. Similar unidirectionally grown $\mathrm{Bi}_{0.5} \mathrm{Sb}_{1.5} \mathrm{Te}_{3}$ single crystals were deformed under much higher pressure (1 GPa) and re-pressed at room temperature by Grasso et al. [173]. They reported a 4fold improvement in the thermoelectric power factor of the forged sample perpendicular to the press direction. An interesting feature of this sample is a very low room-temperature thermal conductivity of $0.34 \mathrm{Wm}^{-1} \mathrm{~K}^{-1}$, which is attributed to high-density of structural defects and stacking disorder. Similar thermopower anisotropy factor to that of the un-pressed single crystal was observed. However, electrical and thermal conductivity exhibit stronger anisotropy. Very recently, Jiang et al. [174] reported a 65\% improvement in the power factor (at 320K) of the hotforged nanostructured bulk $p$-type $\mathrm{Bi}_{0.5} \mathrm{Sb}_{1.5} \mathrm{Te}_{3}$ samples (perpendicular to the press direction) which resulted in a 50\% enhancement in ZT (from 1 to above 1.5). However, the improvement in 
the power factor originates from an unexpected enhancement in the thermopower and not the electrical conductivity ( $\sigma$ actually decreases for the hot-forged sample). The authors correlate this observation to the possible role of point and planar defects created via hot-forging. The unexpected behavior of thermopower is an interesting common feature of several of the recent reports on hot-forged $\mathrm{Bi}_{0.5} \mathrm{Sb}_{1.5} \mathrm{Te}_{3}$ which needs to be further investigated. Deformation-induced donor-like effects in bismuth-telluride-based alloys [175], which can significantly change the carrier concentration, have been recently investigated in detail by $\mathrm{Hu}$ et al. [176,177] and would help explain to some extent the observed behavior of thermopower in the hot-deformed samples. Interestingly, these samples exhibit a relatively small degree of preferred orientation. Figure 15 compares the maximum power factor values reported and discussed above for different $\left(\mathrm{Bi}_{\mathrm{x}} \mathrm{Sb}_{1-}\right.$ х) $)_{2} \mathrm{Te}_{3}$ samples as a function of orientation factor, $F$. It is observed orientation factors close to 1 (corresponding to that of a single crystal in the preferred direction) are not required in order to achieve similar power factor values in the corresponding poly-crystalline sample. Values above 1 $\mathrm{Wm}^{-1} \mathrm{~K}^{-1}$ can be achieved with $\mathrm{F}>0.3$.

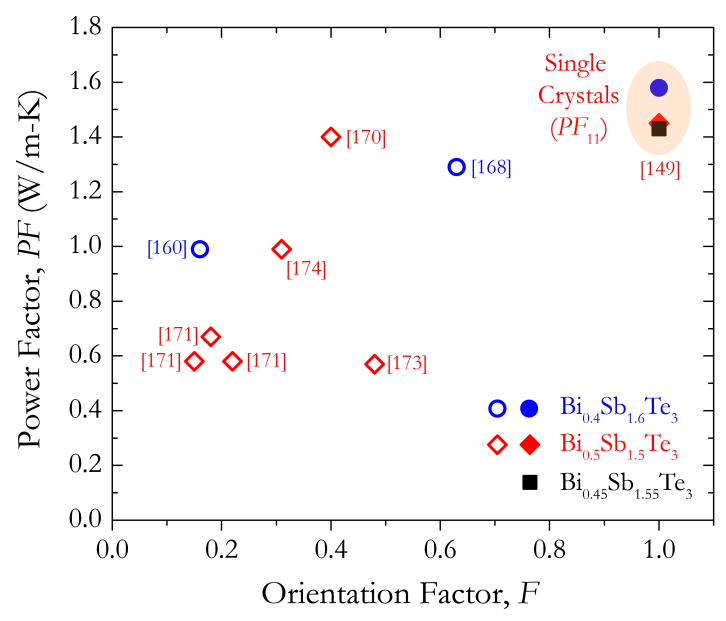

Figure 15 - Reported maximum power factor values for preferentially aligned polycrystalline $\left(\mathrm{Bi}_{\mathrm{x}} \mathrm{Sb}_{1-\mathrm{x}}\right)_{2} \mathrm{Te}_{3}$ samples $(\mathrm{empty}$ symbols)[160,168,170,171,173,174] as a function of orientation factor. The values reported for single crystalline counterparts (filled symbols) in 11 directions (i.e. parallel to the cleavage planes) are also shown. [149]

Similar significant improvements were achieved for the hot-forged $n$-type $\mathrm{Bi}_{2}\left(\mathrm{Te}_{1-\mathrm{x}} \mathrm{Se}_{\mathrm{x}}\right)_{3}$ samples. By preferentially aligning the ab-planes of the crystallites in $n$-type polycrystalline $\mathrm{Bi}_{2} \mathrm{Te}_{2.7} \mathrm{Se}_{0.3}$ using hot-forging, Yan et al.[23] reported a $22 \%$ improvement in the figure of merit $\left(Z T_{\max } \sim 1.05\right.$ at $\left.400 \mathrm{~K}\right)$, which wa was achieved as a result of much improved electrical conductivity (by a factor of 2.3 at room temperature) and therefore power factor in the in-plane direction, in agreement with anisotropy in the corresponding single crystal. Other groups have also investigated $n$-type $\mathrm{Bi}_{2}\left(\mathrm{Te}_{1-\mathrm{x}} \mathrm{Se}_{\mathrm{x}}\right)_{3}$ by hot deformation. [178,179]

Improvement in the electrical conductivity of large-grain $\mathrm{Ca}_{3} \mathrm{Co}_{4} \mathrm{O}_{9}$ samples has also been reported for hot-forged ceramics parallel to the pressed-plane. However, moderate in-plane versus out-of-plane anisotropy factors for the highly-textured samples [180,181,182] are not comparable to values reported for the corresponding single crystal. Nevertheless, this improvement results in $20-30 \%$ enhancement in the power factor over the whole temperature range. 
High magnetic field alignment is another technique which is employed to prepare highlytextured samples from materials possessing anisotropic magnetic susceptibility. In this method the crystallites of materials with feeble magnetic properties are initially aligned in a slurry or colloidal solution. The aligned slurry is then consolidated using spark plasma sintering or hot press. Anisotropic magnetization behavior of $\mathrm{Ca}_{3} \mathrm{Co}_{4} \mathrm{O}_{9}$ single crystals $\left(\chi_{c}>\chi_{\mathrm{ab}}\right)$ reported by Masset et al. [183] motivated the magnetic-field alignment investigations. Horii et al. [184] reported a strong c-axis orientation $(\mathrm{F}>0.96)$ of the $\mathrm{Ca}_{3} \mathrm{Co}_{4} \mathrm{O}_{9}$ crystallites under a magnetic field of 3T which resulted in a 2-fold enhancement in in-plane electrical conductivity (perpendicular to the direction of magnetic field) in the magnetically-aligned sample comparing to the randomly-oriented SPSed sample, while exhibiting relatively similar thermopower values (anisotropy factor $=1.25$ ). Zhou et al. [185] performed a very similar experiment and achieved a similar highly-aligned microstructure $(\mathrm{F}=0.92)$ possessing two-times larger power factor than the non-aligned sample for the whole temperature range. Effect of magnetic field on the degree of grain alignment and the anisotropic transport properties were investigated by Huang et al. [186] A monotonic increase in the electrical conductivity is observed with an increase in magnetic field intensity to $8 \mathrm{~T}$. The power factor along basal plane of this sample is enhanced by about 1.8 times compared to the sample with $0 \mathrm{~T}$. Kuribayashi et al. [187] reported a significant improvement in the thermoelectric properties of magnetically-textured $\mathrm{Bi}_{2} \mathrm{Te}_{3}$ under 10T. The magnetic susceptibilities of $\mathrm{Bi}_{2} \mathrm{Te}_{3}$ in the ab-axis and the c-axis are $\chi_{\mathrm{ab}}=-3.1 \times 10^{6}$ and $\chi_{\mathrm{c}}=-5.5 \mathrm{x}$ $10^{6}$, respectively. A large thermoelectric power factor of $1.48 \mathrm{Wm}^{-1} \mathrm{~K}^{-1}$ at $323 \mathrm{~K}$, similar to reported values reported single crystals, was achieved for highly-textured $\mathrm{Bi}_{2} \mathrm{Te}_{3}$. Kaga et al. [188] reported $35 \%$ improvement in the power factor for c-axis oriented $\mathrm{MnSi}_{1.73}$ (1 micron average grain size) using a $2 \mathrm{~T}$ magnetic field. Very high degree of texturing $(\mathrm{F}>0.9)$ is a common feature of most reports on magnetically-aligned samples. However, the applications of this method are limited to the magnetic properties of material as well as the intensity of magnetic field used to align the crystallites.

Another non-mechanical technique for tailoring microstructure to produce highly-oriented grains in a material is reactive-templated grain growth (RTGG) [189]. This method includes the in-situ epitaxial and/or topotaxial formation of a product material on or in oriented template particles with an anisotropic shape, host-to-guest reaction, and successive grain growth, leading to a single phase grain-oriented polycrystal. Synthesis of highly-textured c-axis oriented $\mathrm{NaCo}_{2} \mathrm{O}_{4}$ ceramics from plate-like $\mathrm{Co}_{3} \mathrm{O}_{4}$ as reactive template is reported by Tajima et al. [190]. Similar investigations were performed on calcium cobaltite thermoelcetrics. [191]

In an excellent review paper focusing on interfaces in bulk thermoelectric materials, Medlin and Snyder [5] raise an important point on the impact of the distribution of the grain boundary types (i.e. tilt or twist boundaries) and its correlation with grain morphology on the electronic and thermal transport properties of strongly textured materials. Materials with columnar grains that are aligned with the texture axis will have a grain boundary population that is inclined toward tilt boundaries. Extruded $\mathrm{Bi}_{2} \mathrm{Te}_{3}$-based materials, which tend to have their directions and their interface planes aligned parallel with the extrusion axis, are examples of such materials. In contrary, materials with plate-like grains aligned perpendicular to the texture axis will have a grain boundary distribution biased towards twist boundaries. $\mathrm{Bi}_{2} \mathrm{Te}_{3}$ and the cobaltites are examples of such materials. Due to the fundamentally different interfacial defect structure in these two types of interface, differences in the electronic and thermal transport properties might 
be expected. Therefore, more in-depth microstructural studies would help to clarify the role of the interfaces on the transport behavior. [5] The work by Heinz et al. [192] applying quantitative microstructural control in advanced composites is along this direction.

In conclusion, as it was observed significant improvements in the thermoelectric power factor and thermoelectric performance of anisotropic polycrystalline materials can be achieved through texture engineering in conjunction with nanostructuring. Recent intriguing results on hot-forged thermoelectrics would certainly trigger more discussions and investigations as deeper understanding of the character of defects and interfaces in the improvement observed in highly oriented samples is needed.

\subsubsection{Composite engineering}

The idea of discovering new properties or improving the existing properties in a multicomponent system over those of the individual components is the heart of composite engineering. In case of bulk thermoelectric materials, the ultimate goal is to improve the thermoelectric figure-of-merit over that of the constituents. Initial theoretical attempts to understand the thermoelectric properties of composite materials were performed by Herring[193], Webman[194], and Straley[195] focusing on thermopower in inhomogenous materials. A decade later, Bergman and Levy[196] developed an effective-medium theory based on linearized thermoelectric transport equations to explain the thermoelectric properties of a twocomponent composite mixture. They concluded that the effective figure-of-merit, $\mathrm{ZT}_{\text {effective, }}$ of a two-component composite can never be greater than its largest value among its constituting components. However, in a follow-up paper, Bergman and Fel[197] showed that sometimes the effective power factor of a composite mixture can be greater than the power factors of each component. Heremans and Jaworski[198] experimentally validated Bergman and Fel calculations for the micron-sized bismuth-silver composite system. However, the limitations of the Bergman and colleagues' theorem need to be considered before making generalizations about the model. It should be noted that the $\mathrm{ZT}_{\text {effective }}$ concept is valid only when the medium is homogeneous on the length scales of the thermoelectric legs and in the absence of the crystallite size effects and charge transfer from boundaries (i.e. boundary scattering). Therefore, the concept cannot be applied to explain the overall thermoelectric properties if the composite material is graded (segmented) or whether it is inhomogeneous in nanoscale. It is known that phonons and charge transport are influenced by crystallite boundary scattering especially when the size of the crystallites becomes comparable to electrons and phonons characteristic lengths. Therefore, the ZT is expected to be improved under such conditions that the crystallite size is small compared to the phonon mean free path and large compared to the charge carriers energy relaxation lengths. [199,200] Figure 16 schematically shows the desired behavior of the temperature dependence of electrical conductivity and thermopower (above room temperature) of a twocomponent composite and its individual components. Ideally, the maximum increase in the electrical conductivity is desired with minimum (or no) drop in the Seebeck coefficient of the highest component. 
(a)

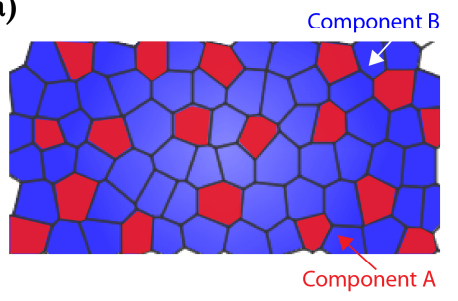

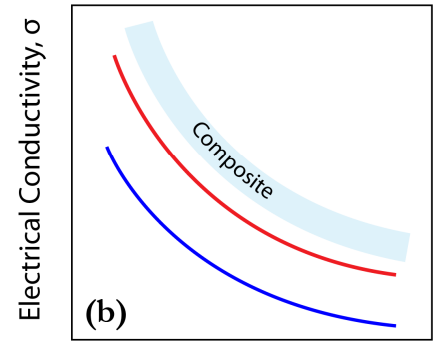

Temperature, $T$

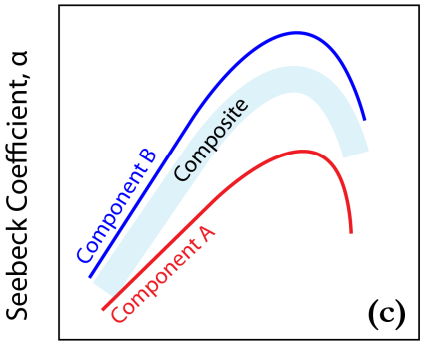

Temperature, $T$

Figure 16 - (a) Schematic of a two-component composite. Component A possesses higher electrical conductivity and lower Seebeck coefficient than the component B (matrix). (b) Schematic electrical conductivity behavior as a function of temperature for the individual components and the improvement expected for the composite. (c) Corresponding schematic Seebeck coefficient as a function of temperature for the individual components and that of the composite. The extent of increase in the electrical conductivity and the drop in Seebeck coefficient over that of the best component depends strongly on the characteristic lengths such as crystallite lengths of the components, phonon and carrier mean free path as well as energy relaxation length.

The thermoelectric power factor in nanowire-composite matrix structures was theoretically studied by Broido and Mingo.[201] They found that PF can be significantly enhanced compared to the bulk material. However, this enhancement is significantly dependent on carrier effectivemass, height of the confining potential barrier, and the cross-sectional size of the nanowirecomposite matrix. Neophutou et al.[202] showed that the power factor can be enhanced in heavily-doped two-phase nanocomposite materials that are doped inhomogeneously. They predicted an improvement in the power factor by almost an order of magnitude for inhomogeneous p-type $\mathrm{Si}$ nanocomposite $(30 \mathrm{~nm}$ grain size $)$ versus the corresponding homogeneously doped bulk Si.

Bian and Shakouri,[203] and Bian et al.[204] explored the possibility of improvement in graded composites by focusing on the maximum cooling temperature and efficiency rather than average ZT. They showed that the maximum cooling of graded legs, e.g. standard $\mathrm{Bi}_{2} \mathrm{Te}_{3}$, can be improved by $27 \%$, thanks to the redistribution of Joule heating, by reducing the doping in $1 / 4$ the leg near the hot junction. The possibility of improvement of both maximum cooling and efficiency at large temperature differences was shown, even though the average ZT of the leg is

reduced by $\sim 10 \%$. The idea of improving the efficiency through inhomogeneous doping was

first theoretically discussed by Mahan[205]. Recently, Yang et al.[206] revisited Bergman and Levy's calculations under nonlinearized coupling and concluded that conversion efficiency of a 
bilayer graded thermoelectric composite is not bound by its constituents and can be larger than those of its components in the absence of size and interface effects.

In recent years, the theoretical calculations discussed above have been implemented experimentally in combination with nanostructuring effects to improve the thermoelectric power factor and the overall ZT of a wide range of composite materials. Zamanipour and Vashaee [207] reported $\sim 20 \%$ improvement (at $830^{\circ} \mathrm{C}$ ) in the power factor of $\mathrm{SiGe}$ by adding $\mathrm{CrSi}_{2}$ nanoinclusions. Peng et al. [208] reported a significant improvement in the power factor of $\mathrm{Yb}_{0.2} \mathrm{Co}_{4} \mathrm{Sb}_{12} / 6 \mathrm{wt} \% \mathrm{AgSbTe}_{2}$ nanocomposites by a factor of 7 at $300 \mathrm{~K}$ over that of the $\mathrm{Yb}_{0.2} \mathrm{Co}_{4} \mathrm{Sb}_{12}$ matrix and a factor of 5 over the reported value for single phase $\mathrm{AgSbTe}_{2}$ [209]. They showed that for $8 \mathrm{wt} \% \mathrm{AgSbTe}_{2}$ there is a crossover where the sample behaves like a Bergman's composite due to the agglomeration of $\mathrm{AgSbTe}_{2}$ phase. This improvement in the power factor combined with the reduction in the thermal conductivity due to $\mathrm{AgSbTe}_{2}$ nanoinclusions $(<100 \mathrm{~nm}$ ), resulted in an improvement in ZT by a factor of 4 at $300 \mathrm{~K}$ over that of the $\mathrm{Yb}_{0.2} \mathrm{Co}_{4} \mathrm{Sb}_{12}$ matrix. Improvement in the power factor and $\mathrm{ZT}$ of the modulation-doped $\mathrm{SiGe}$ in $\mathrm{SiGe} /$ heavily-doped $\mathrm{Si}(20-50 \mathrm{~nm})$ nanocomposites discussed earlier can also be thought of as a special case of composite engineering involving charge transfer effects. Recently, several groups reported improvement in the thermoelectric properties of multi-component oxide composites.[24,210,211] Dehkordi et al.[24] reported a significant improvement in the power factor (> 70\% at $775 \mathrm{~K}$ ) of bulk polycrystalline $\mathrm{SrTiO}_{3}$ ceramics by non-uniform Pr doping (i.e. Pr-rich grain boundary/Pr-poor grain composite). This marked enhancement originated from a marked improvement in the carrier mobility, a factor of 2 at room temperature. This resulted in a $30 \%$ improvement in the figure-of-merit for the whole temperature range over all previously reported values for doped single- and polycrystalline $\mathrm{SrTiO}_{3}$ ceramics.[212] Such enhancement in PF could not be explained with effective medium theory and a charge transfer mechanism is suspected to be involved.

One common feature of the majority of the experimental reports on the composite thermoelectrics is a broader maximum power factor peak with composite engineering, which would result in a broader ZT peak and higher average ZT. The PF and the ZT of a multiple-phase composite tend to exhibit an effective behavior that is averaged over the TE properties of each constituent and of the interfaces (in the context of effective medium approximation). Unless some resonance occurs, the ZT peak of a composite tends to be broad. Recently, a broad temperature plateau for $\mathrm{ZT}>2$ was reported in phase-separated $\mathrm{PbTe}_{0.7} \mathrm{~S}_{0.3}$ by Wu et al.[213]. These samples are composites of PbTe-rich and $\mathrm{PbS}$-rich region formed by spinoidal decomposition of $\mathrm{PbTe}_{0.7} \mathrm{~S}_{0.3}$.

Motivated by the flexible electronics movement, another avenue which has been explored in the realm of composite thermoelectrics within the past few years is the thermoelectric properties of organic-inorganic composites. In this case, typically the goal is to improve the electrical properties (particularly $\sigma$ ) of the organic matrix via composite engineering. In this direction, power factor value of $\sim 2.1 \times 10^{-2} \mathrm{Wm}^{-1} \mathrm{~K}^{-1}$ were achieved by Zhang et al. [214] for CLEVIOS FE- $\mathrm{T} / \mathrm{Bi}_{2} \mathrm{Te}_{3}$ composites. Relatively large in-plane thermoelectric power factor values of $\sim 4.8 \mathrm{x}$ $10^{-2} \mathrm{Wm}^{-1} \mathrm{~K}^{-1}$ at room temperature were reported by $\mathrm{Yu}$ et al.[215] for carbon nanotube(CNT)based organic composites (60 wt $\%$ CNT/40 wt $\%$ polymers), which are orders of magnitude larger than reported values for typical polymer composites. Recently, Yee et al.[216] reported 
maximum power factor value of $\sim 3.3 \times 10^{-2} \mathrm{Wm}^{-1} \mathrm{~K}^{-1}$ for PEDOT-PSS/Te Nanowire composites at $300 \mathrm{~K}$.

These results prove the potentials of composite engineering in improving the thermoelectric power factor and the figure-or-merit in inorganic and organic materials. However, to date this strategy has been extensively studied experimentally in the context of thermal conductivity reduction. Therefore, there is a huge potential for further experimental explorations and investigations towards improvement of thermoelectric power factor using innovative combination of thermoelectric components in conjunction with optimization of components' characteristic lengths.

\subsection{Strategies to Simultaneously Enhance Electrical Conductivity and Seebeck Coefficient}

\subsubsection{Carrier Pocket Engineering and Convergence of the Electronic Bands}

As it was discussed before, a large number of degenerate bands is highly desired in the electronic structure of a potential thermoelectric material. Many of the known thermoelectrics typically possess $N_{v} \leq 6$ equivalent valleys. Band degeneracy increases when multiple bands have the same or comparable energy within a few $k_{\mathrm{B}} T$ (i.e. they can be effectively thought of as degenerate). This situation can happen (i) when multiple bands possess band extrema with no or insignificant difference in energy (orbital degeneracy), or (ii) when multiple carrier pockets in the Brillouin zone are degenerate due to their symmetrical equivalency (valley degeneracy). A valley degeneracy, $N_{\mathrm{v}}$, increases the density-of-states effective mass, $m^{*}$, by a factor of $N_{v}^{2 / 3}$. Convergence of many charge-carrying valleys (increasing $N_{v}$ ) has no detrimental effects on electronic transport. The carrier mobility is nominally unaffected by $N_{v}$, but there might be some reduction due to intervalley scattering. [8] It should be noted that higher doping levels are required to fully realize the effect of band convergence. This typically leads to a slight increase in the electrical conductivity originating from increased carrier concentration $(\sigma=n e \mu)$.

The concept of engineering the alignment of charge-carrying valleys to improve the thermoelectric power factor was first proposed by Koga et al. [217,218] in the context of low dimensional structures. This idea, which is now known as carrier pocket engineering, is referred to taking advantage of the confinement effects to achieve convergence of symmetrically equivalent bands (i.e. to increase $N_{v}$ ) by tuning the superlattice design parameters such as superlattice period, layer thicknesses of the quantum well and the barrier, the growth direction,, the composition, and/or the lattice constant of the substrate. [219]

The predictions were first made for the case of $\Gamma$-point $\left(N_{v} \approx 1\right)$ electrons for GaAs quantum wells and X-point $\left(N_{v} \approx 3\right)$ electrons in the AlAs barriers in short period GaAs/AlAs quantumwell superlattices. The $\Gamma$ - and L- valleys in bulk GaAs (with $N_{v}=1$ and 4, respectively) and Xvalleys in bulk AlAs (with $\mathrm{N}_{\mathrm{v}}=3$ ) are within $0.28 \mathrm{eV}$ of each other as shown in Figure 17 (a). However, in GaAs/AlAs superlattices, these valleys can be brought much closer together by careful optimization of the structure of superlattice. Figure 17 (c) shows the difference in energies of the valleys as a function of the width of the quantum well. It was found that for (111)-oriented GaAs/AlAs superlattices, the $\Gamma, \mathrm{X}$ and $\mathrm{L}$ valleys lie very close in energy (with an

energy spread of just $0.05 \mathrm{eV}$ ) for $\mathrm{d}_{\mathrm{GaAs}}=\mathrm{d}_{\mathrm{AlAs}}=20 \AA$, and hence, the band degeneracy is 
increased to $N_{v}=8$ (from $N_{v}=1$ for transport via $\Gamma$ valley only). As a result, an enhancement of power factor occurs due to both (i) the local increase in the density-of-states for both pockets, and (ii) the global increase in the density-of-states due to the addition of the extra active valleys, which were previously inactive. [4] A 4-fold increase in the ZT is predicted for the carrierpocket-engineered GaAs/AlAs superlattices due to the addition of $\mathrm{L}$ - and $\mathrm{X}$ - pockets. As it is shown in Figure 17 (d), an optimum $\mathrm{Z}_{3 \mathrm{D}} \mathrm{T}$ was found at $n=5 \times 10^{19} \mathrm{~cm}^{-3}$, almost an order of magnitude larger than the carrier concentration which optimizes the $P F$ and $Z T$ for $\Gamma$-valley transport only. This is a direct consequence of larger $N_{v}$ and more accessible pathways for carriers to participate in charge transport.

The encouraging predictions on GaAs/AlAs system triggered investigations of such sizedependent design process and implementation of model calculations to materials with better thermoelectric properties, such as $\mathrm{Si} / \mathrm{Ge}$ superlatices and $\mathrm{Bi}_{1-\mathrm{x}} \mathrm{Sb}_{\mathrm{x}}$ nanowires. The concept of carrier pocket engineering is applied to strained $\mathrm{Si} / \mathrm{Ge}$ superlattices next to take advantage of the large number of conduction band valleys of $\mathrm{Si}$ and $\mathrm{Ge}\left(N_{v}=6\right.$ and 4 at the $\Delta$ and $\mathrm{L}$ points of the Brillouin zone, respectively). High $\mathrm{ZT}_{3 \mathrm{D}}$ values of 0.24 and 0.96 were predicted at $300 \mathrm{~K}$ for

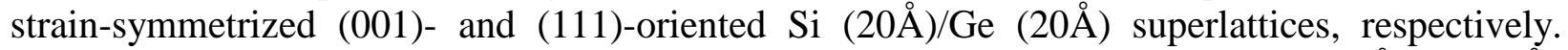
[220,221] Experimental proof-of-principle investigations on (001) oriented $\mathrm{Si}(20 \AA) / \mathrm{Ge}(20 \AA)$ superlattices were performed by Koga et al. [222] and $\mathrm{ZT}_{3 \mathrm{D}}=0.1$ was achieved for (001)oriented $\mathrm{Si}(20 \AA) / \mathrm{Ge}(20 \AA)$ superlattices. Maximum power factor of $P F \sim 0.47 \mathrm{~W} / \mathrm{m}-\mathrm{K}$ was reported for this structure at $n \sim 1.1 \times 10^{19} \mathrm{~cm}^{-3}$. Further improvement of the power factor up to $1.1 \mathrm{~W} / \mathrm{m}-\mathrm{K}$ and above was predicted by improving the structural quality of the superlattices, and introducing the modulation-doping schemes. Application of the concept to $\mathrm{Bi}_{1-\mathrm{x}} \mathrm{Sb}_{\mathrm{x}}$ nanowires also held promise of improvement of thermoelectric power factor and ZT. Rabin et al. [223] predicted an exceptionally high $\mathrm{ZT}$ of 1.2 at $77 \mathrm{~K}$ for $40-\mathrm{nm} \mathrm{Bi}_{0.87} \mathrm{Sb}_{0.13}$ nanowires arising from the convergence of up to 10 valence valleys. 
(a)

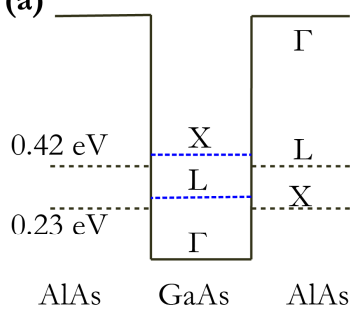

AlAs GaAs AlAs

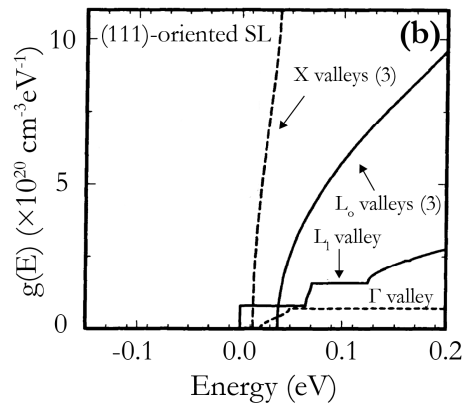

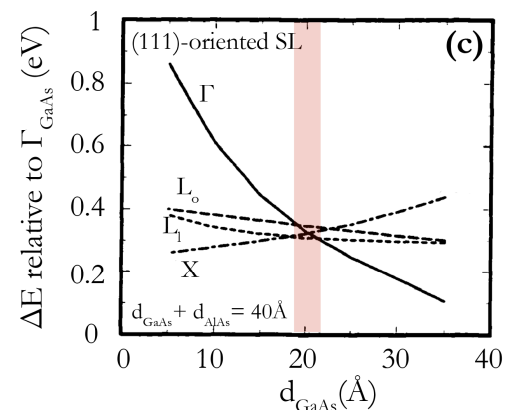

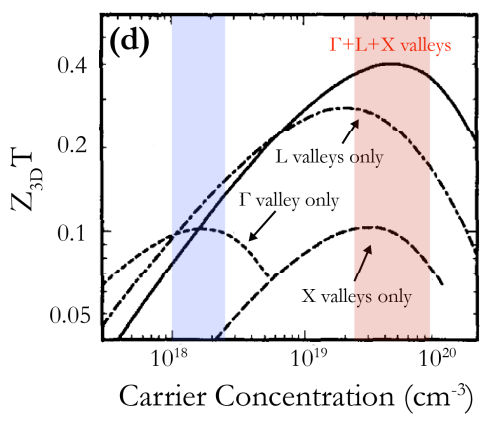

Figure 17 - (a) Conduction band offset at various high symmetry valleys in the Brillouin zone for GaAs/AlAs superlattices, (b)

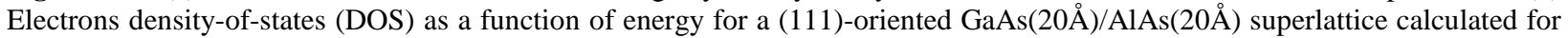
various subbands derived from $\Gamma, \mathrm{X}, \mathrm{L}_{\mathrm{t}}$ and $\mathrm{L}_{\mathrm{o}}$ valleys. The number in the parentheses denotes the number of equivalent valleys that are degenerate in the energy, (c) Calculated energies for various subband edges in a (111)-oriented GaAs/AlAs superlattice, measured from the conduction band edge at the $\Gamma$ point, and (d) Calculated $\mathrm{Z}_{3 \mathrm{D}} \mathrm{T}$ values for a (111)-oriented $\operatorname{GaAs}(20 \AA) / \operatorname{AlAs}(20 \AA)$ superlattice as a function of carrier concentration considering $\Gamma$, L and $\mathrm{X}$ valleys separately, as well as treating all three valleys together (solid line). Adapted from Ref. [224].

Increasing the convergence of valleys in a bulk material via band engineering was first demonstrated by Pei et al. [33], which triggered follow-up investigations. They showed that by tuning the doping and composition of the $\mathrm{PbTe}_{1-\mathrm{x}} \mathrm{Se}_{\mathrm{x}}$ solid solution, a convergence of at least 12 valleys in the valence band is achieved at high temperatures $\left(\mathrm{T}>450^{\circ} \mathrm{C}\right)$ leading to a maximum power factor of $2.08 \mathrm{Wm}^{-1} \mathrm{~K}^{-1}$ at $800 \mathrm{~K}$ for bulk $\mathrm{PbTe}_{0.85} \mathrm{Se}_{0.15}$, the largest reported for p-type $\mathrm{PbTe}$ at this temperature. The valence band maximum in PbTe occurs at the $\mathrm{L}$ point in the Brillouin zone (referred to as the L band), where the valley degeneracy, $N_{v}$, is 4 . However, there exists a second valence band $0.2 \mathrm{eV}$ below the $\mathrm{L}$ band, along $\Sigma$ line (referred to as the $\Sigma$ band) which has a valley degeneracy of 12 (See Figure 17(a)). Pei et al. showed that by appropriately doping the $\mathrm{PbTe}_{1-\mathrm{x}} \mathrm{Se}_{\mathrm{x}}$ solid solution and engineering the band alignment at the desired temperature, a valley degeneracy of 16 can be achieved where the $\mathrm{L}$ and $\Sigma$ bands meet. $\mathrm{L}$ and $\Sigma$ band-crossing as a function of temperature is shown schematically in Figure 17 (b). As it is expected, the calculated optimal doping concentration for multi-valley transport is almost an order of magnitude larger than calculated by Fritts for single-valley transport (see Figure 18 (c)).[225] 
(a)

(c)
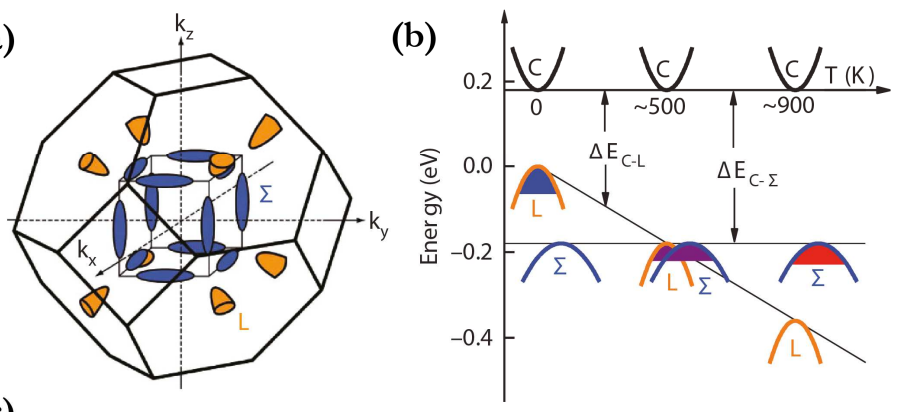

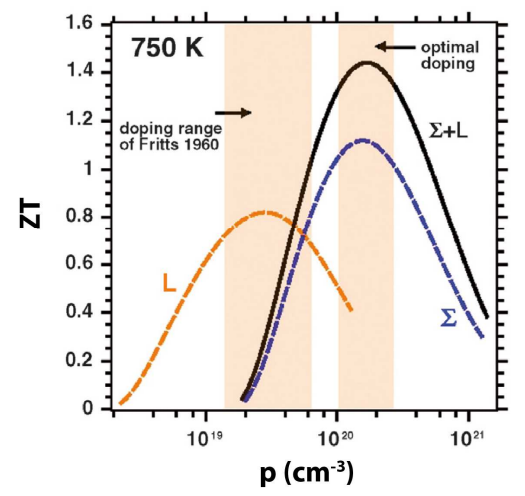

Figure 18 - (a) Brillouin zone of $\mathrm{PbTe}_{1-\mathrm{x}} \mathrm{Se}_{\mathrm{x}}$ with 8 hole half-pockets (orange), i.e. $\mathrm{N}_{\mathrm{v}}=4$, at the $\mathrm{L}$ point and 12 hole pockets (blue), i.e. $\mathrm{N}_{\mathrm{v}}=12$ along the $\Sigma$ line. (b) Schematic diagram of the valence band edges in $\mathrm{PbTe}_{0.85} \mathrm{Se}_{0.15}$ as a function of temperature. $\mathrm{L}$ and $\Sigma$ bands converge at $500 \mathrm{~K}$, resulting in multivalley transport. Reprinted with permission from Nature publishing group from Ref. [33]. (c) The calculated $\mathrm{ZT}$ at $750 \mathrm{~K}$ as a function of carrier concentration for $p$-type PbTe for transport from the $\mathrm{L}$ or $\Sigma$ band alone or " $\Sigma+\mathrm{L}$ ". A significant increase of peak ZT is observed for multivalley transport, which can only be realized at carrier concentrations twice that reported by Fritts. Reprinted with permission from Elsevier Ltd. from Ref. [225].

Follow-up studies by Pei et al. investigated further engineering of PbTe band structure through alloying with other potential binary compounds such as MgTe[226], CdTe[227], and MnTe[228]. Reported power factor is shown as a function of temperature in Figure. Very similar transport behavior was observed for these three pseudo-binary solutions. The room temperature band gap increases with increasing $\mathrm{M}$ content in $\mathrm{Pb}_{1-\mathrm{x}} \mathrm{M}_{\mathrm{x}} \mathrm{Te}(\mathrm{M}=\mathrm{Cd}, \mathrm{Mg}$, or $\mathrm{Mn})$, in such a way that the energy of the light valence band is reduced to achieve an effective alignment with the heavy band even at room temperature. This is an opposite effect to the $\mathrm{PbTe}_{1-\mathrm{x}} \mathrm{Se}_{\mathrm{x}}$ alloys with a slightly lower band gap that is aligned at higher temperatures. The increased band gap has the effect of shifting the detrimental contribution of thermally activated minority carriers to slightly higher temperatures. However, the increased $\mathrm{m}_{\mathrm{b}} *$ of the light valence band due to the widening of the band gap by alloying significantly reduces $\mu_{\mathrm{H}}$ and therefore the power factor in Na-doped $\mathrm{Pb}_{1 \text { - }}$ ${ }_{x} M_{x} T e(M=C d, M g$, or $\mathrm{Mn})$ with $n_{H}<1 \times 10^{19} \mathrm{~cm}^{-3}$. In this carrier-concentration range, the transport properties are dictated by the light band. However, an improvement in the power factor can be achieved due to an effective increase of $N_{v}$ in the alloy when $\mathrm{n}_{\mathrm{H}}<5 \times 10^{19} \mathrm{~cm}^{-3}$. It is observed that larger decrease in the carrier mobility in the alloys, lead to similar values of power factor to PbTe:Na. However, the significant reduction in the thermal conductivity originating from alloy scattering results in $\sim 30 \%$ higher average ZT for the pseudo-binary alloys. 
Zhao et al.[229] revisited Pei et al. investigations on PbTe-MgTe recently in order to take advantage of the solid solubility issue to form in-situ nanostructures to further reduce the thermal conductivity. It is worth mentioning that Zhao et al. reported a rather different behavior for $\mathrm{PbTe}-\mathrm{MgTe}$ solid solution (as is reflected in the temperature-dependence of the power factor in Figure 19).

Recently, Saleh [230] reported an enhancement in the power factor of PbTe-SnTe alloys for samples prepared using melting-quenching-annealing method. Maximum value of $0.6 \mathrm{~W} / \mathrm{m}-\mathrm{K}$ was achieved at room temperature for samples with 4 mol\% SnTe.

Equally important to the band structure engineering predictions is the phase diagram and solid solubility considerations which dictates the extent to which the potential of band engineering can be exploited in reality. MgTe was found to be the only group II telluride with substantial solubility in $\mathrm{PbTe}$. PbTe-PbSe binary is the only solution with significant solubility and possessing favorable characteristics.

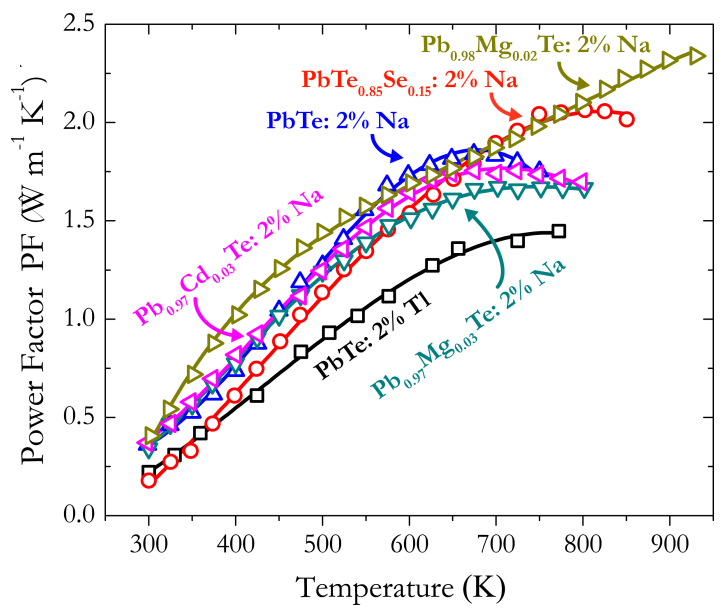

Figure 19 - Temperature-dependence of thermoelectric power factor for p-type PbTe samples. [33,37,66,226,227,229]

Band-convergence concept has also been implemented on $n$-type bulk polycrystalline $\mathrm{Sb}$-doped $\mathrm{Mg}_{2} \mathrm{Si}_{1-\mathrm{x}} \mathrm{Sn}_{\mathrm{x}}$ solid solutions by Zaitsev et al. [231,232] (prior to Pei et al.'s work), and recently by Liu et al.[25] Formation of solid solution of appropriate composition $(x \approx 0.6-0.7)$ allows for the alignment of the two band extrema, which results in an enhanced density-of-states effective mass, and hence, a significant improvement in the thermoelectric power factor. Recently, this concept has been reported for solid solutions of $p$-type SnTe with CdTe[233], SnSe[234], and very recently $\mathrm{HgTe}[235]$. Maximum power factor of $2.5 \mathrm{~W} / \mathrm{m}-\mathrm{K}$ was achieved for $2 \mathrm{~mol} \% \mathrm{Bi}$ doped $\mathrm{SnTe}$ alloyed with $3 \mathrm{~mol} \% \mathrm{HgTe}$, which is the highest reported for the $\mathrm{SnTe}$ material system.

In the light of these findings, very recently, Chen et al.[38] reinvestigated the origin of high thermoelectric power factor $\left(2.45 \mathrm{Wm}^{-1} \mathrm{~K}^{-1}\right.$ at $\left.700 \mathrm{~K}\right)$ of $p$-type $\left(\mathrm{Ag}_{\mathrm{x}} \mathrm{SbTe}_{\mathrm{x} / 2+1.5}\right)_{15}(\mathrm{GeTe})_{85}$ (TAGS) thermoelectric materials with $0.4 \leq \mathrm{x} \leq 1.2$. It was concluded that large density-ofstates effective mass in these compounds originates from the large band mass of the primary valence band as well as the high degeneracy of pockets in the Fermi surface from the second 
valence band. Calculations suggest the involvement of $N_{v} \sim 16$ valleys in transport for $p>9 \mathrm{x}$ $10^{20} \mathrm{~cm}^{-3}$. The highly degenerate Fermi surface increases the total density-of-states without decreasing mobility.

Engineering the electronic band convergence stimulated by doping or alloying is therefore proven to be an effective strategy and a new promising avenue for optimizing properties of thermoelectric materials forming solid solutions.

\subsubsection{Invisible Dopants}

We emphasized in section 3.1 (modulation doping) that the thermoelectric materials are heavily doped and therefore ionized impurity scattering in many cases, results in strong suppression of carrier mobility. Dopants are added intentionally to the thermoelectric semiconductors to provide the high level of carrier concentration required in these materials. By engineering the introduced doping centers (e.g. by modifying their shape, size, chemical potential and material type) one can design doping centers, which minimally scatter conduction carriers and therefore can suppress the deleterious effect of doping.

The possibility of adding invisible dopants to enhance the thermoelectric power factor was inspired by two observations. The first observation was the Ramsauer-Townsend (RT) effect [236,237]. Ramsauer and Townsend observed that for slow-moving electrons in noble gases, such as argon, krypton, or xenon, the probability of collision between the electrons and gas atoms shows a minimum value for electrons with a certain amount of kinetic energy (about 0.7 $\mathrm{eV}$ for xenon gas). Zebarjadi et al. [238] transferred the same idea to the thermoelectric materials. They designed particular core-shell nanoparticles that could donate carriers to the host matrix and at the same time, the resulted ionized core-shell nanoparticle shows an anti-resonance scattering profile. In this design, electrons of a narrow energy range (cloaking window) observe a much smaller scattering cross section (up to 4 orders of magnitude) compared to the physical cross section of the nanoparticles $\left(\pi \mathrm{a}^{2}\right)$. Since only electrons with energies in the Fermi window $\left(E_{F} \pm\right.$ few $\left.k_{B} T\right)$ contribute to the transport, by overlapping the Fermi window and the cloaking window, one can assure that the doping centers are invisible to all conduction electrons.

The second inspiration for designing invisible dopants is the advances in optical cloaking. In the optical cloaking analogy, an object is hidden from a range of light wavelengths [239,240,241]. Similarly, electrons have wave-like behavior and if optical cloaking is possible, electronic cloaking should be possible. Two groups have demonstrated the feasibility of this idea recently by designing artificial core-shell nanoparticles [242,243]. In a more recent publication, Shen et al. [244] extended this idea to design realistic hollow nanoparticles. They used a combinatorial algorithm to search a large class of materials and identified several candidates to demonstrate electronic cloaking. 


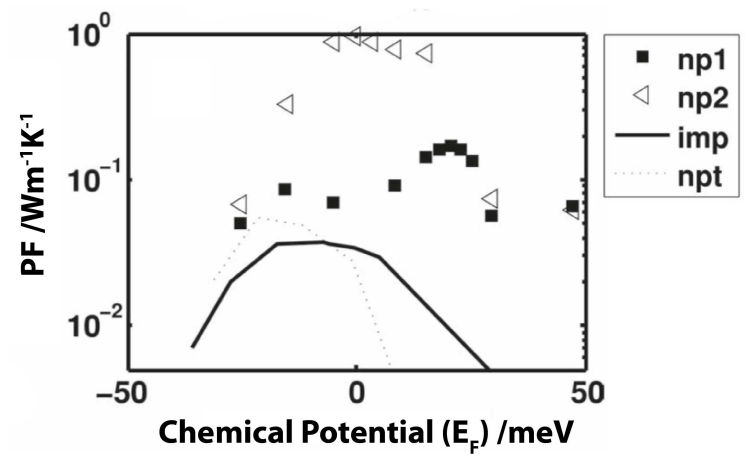

Figure 20 - The chemical potential dependence of the power factor calculated for doped GaAs at $\mathrm{T}=50 \mathrm{~K}$. Four different types of dopants are considered: regular impurities (solid line, imp), traditional nanoparticles (dotted line, npt), and two sets of antiresonant nanoparticles ( $\mathrm{np} 1$ and $\mathrm{np} 2$ ). The considered traditional nanoparticle is a one layer nanoparticle made out of the core of the nanoparticle denoted as np2. Reproduced with permission from WILEY-VCH Verlag from Ref. [238]

There has not been any experimental demonstration of electronic cloaking so far. The main challenges are the small sizes of the designed nanoparticles (limited to few nanometers) and the required size uniformity of such particles. Large predicted thermoelectric power factors calculated for ideal cases of uniform samples are however very encouraging. More theoretical investigations are needed to confirm that the enhancements are still possible once size variations, incoherent and multi-scatterings are included.

\subsubsection{Interfacial Effects}

Above we have discussed two promising energy dependent scattering mechanisms toward enhancing the PF. Compared to the Resonant Levels (RLs) mechanism, the electron energy filtering scheme is more viable to be implemented in TE material, interfaces are arguably the most proper place for such implementation. The rationale behind this argument is straightforward yet subtle. Most state-of-the-art TE materials used in large-scale application are polycrystalline for the sake of cost-efficiency. Polycrystalline material is featured by a multitude of interfaces (grain boundaries), in particular, the interfaces in nanocomposite are so large that they must be treated as an individual "phase" toward enhancing the $P F$. Not only that, in the context of effective medium approximation, the TE performance of a composite cannot exceed that of the best performing constituent if without contribution from interfaces (grain boundaries)[196,197] or charge/mass transfer[143,22]. In general, interfaces can be utilized to decouple the otherwise adversely inter-dependent resistivity, thermopower and thermal conductivity in single-phased bulk material $[5,245,246,247,248]$. To help elucidate the interfacial effect toward enhancing the $P F$, it is instructive to first clarify the relation between "size effect" and "interfacial effect".

We limit our discussion mainly on the charge carriers in the following as the main theme of this paper is the $P F$. In TE study, there are two fundamental types of size effect: quantum size effect and classical size effect, they are associated with a fundamental length scale, the wavelength and the mean free path, respectively. The quantum size effect arises from the confinement-induced changes in the electron band structure (i.e., frequency-wavelength dispersion relation), especially 
the position of Fermi level, size of the band gap, the density of states, and the effective mass. Confinement effect becomes important when the sample size is comparable to the electron's de Broglie wavelength. On the other hand, the classical size effect is concerned with the limitation of the carrier mean free path of electron due to scattering in transport, e.g. interface scattering, which is a key scattering mechanism in polycrystalline materials and nanocomposites. Although it is not always easy to experimentally differentiate the quantum and classical size effect, both effects can enhance the $P F$ via optimizing the band structure and the scattering mechanism. In this regard, the size effect impacts the $P F$ in a similar manner how the RLs mechanism impacts the $P F$. The quantum size effect on the $P F$ was discussed in section 3.1.1 above.

In order to enhance the $P F$, interfaces must undertake multi-tier preferential scattering:

(i) Interfaces should scatter phonons more effectively than electrons. In the context of defect physics, interface is a planar ("2-dimensional") defect with its characteristic length scale on the order of $\mathrm{nm}$ to $\mathrm{mm}$. Hence interface is more effective in scattering longer mean-free-path electrons and phonons. Most TE materials are heavily substitutionally doped in which the electron mean free path is much shorter than the typical grain size (on the order of the spacing between two opposite grain boundaries). In addition, phonons possess a temperature dependent spectrum wide in energy and momentum frequency. As a result, the effect of interface scattering is usually important only in the lattice thermal conductivity at low temperatures and gets marginal at elevated temperatures.

Nevertheless, the interfacial scattering is especially useful when the phonon mean free path is longer than the electron counterpart, in this case one can manage proper grain size to scatter phonons more than electrons without seriously degrading the electrical transport [249]. In case grain size cannot be used as a control parameter, interfacial roughness is an alternative. One can control the interfacial roughness, somewhat independent of grain size, to preferentially scatter phonons without much adversely affecting the $P F[250,251]$. One can also fabricated a thermoelectrically favorable interface (i.e., nanocoating or nanoplating processing), for example, in the hydrothermally treated $\mathrm{Bi}_{2} \mathrm{Te}_{3}, \mathrm{PbTe}, \mathrm{CoSb}_{3}$ systems [252,253,254]. It is commonly accepted that a coherent or semi-coherent (epitaxial-like) grain boundary is necessary to retain electrical properties [72,73] while the total interfacial area per volume governs the phononic thermal conductivity [255].

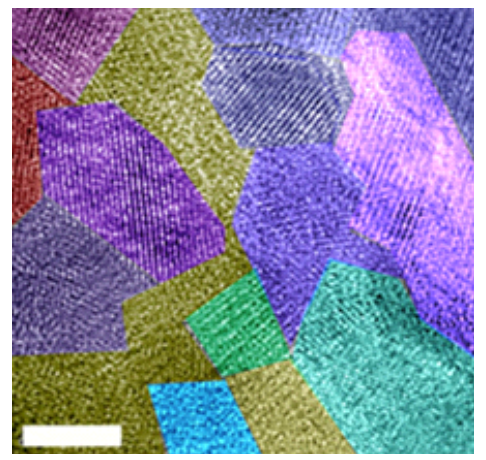

Figure 21 - A high resolution transmission electron microscope image taken on melt-spun-spark-plasma sintered $p$ - $\mathrm{Bi}_{2} \mathrm{Te}_{3}$ shows many nanocrystals (in pseudo-color) with coherent or semi-coherent grain boundaries. The scale bar is $10 \mathrm{~nm}$. (unpublished) 
(ii) Interfaces scatter those carriers with lower mean energy more effectively than those with higher mean energy in line with the electron energy filtering mechanism (c.f. section 3.1.2).

(iii) Interfacial charged defects scatter minority carriers more effectively than majority carriers. Concerning the effect on the electrical transport, interfacial charge defect are not a simple superposition of a charged point ("0-dimesnional") defect and a charge neutral ("2-dimensional") grain boundary. Recent studies suggested that the interfacial charged defects not only tune the carrier concentration within the grain (and thus suppress the detrimental bipolar effect) but also act as potential barriers that scatter minority carriers more effectively than majority carriers. This was inferred from the observations in the ball-milled-hot-pressed $p$-type $\mathrm{Bi}_{2} \mathrm{Te}_{3}$ [36] and the chemically-exfoliated-spark-plasma-sintered $n$-type $\mathrm{Bi}_{2} \mathrm{Te}_{3}$ [256]. It was recently found that the spark plasma sintering process, especially the ON-OFF ratio of the square pulse current, could be utilized to selectively modify the grain boundaries in $\mathrm{Bi}$. As a result, all three individual TE properties are improved. However, the underlying mechanism is yet fully understood, in particular, as to whether the observation is associated to the surface states of Bi [257]. So far, we are still trying to better understand and control such interfacial effects toward enhancing the $P F$.

\section{Conclusions and Future Perspective}

The global demand for sustainable energy and the environmental concern on the use of fossil fuel have imposed an impressing need for the advancement of alternative energy conversion technologies, including thermoelectrics. The modular aspects of thermoelectric devices, all solid state, feasibility for miniaturization, no greenhouse gas emission, no rotation parts, ease to be used in tandem with other alternative energy conversion technologies, guarantee a position in our solution package to the energy challenge in the $21^{\text {st }}$ century.

On the road to improve the thermoelectric materials performance, we have established in the past 10-15 years an arsenal of technical tools and ways to achieve low lattice thermal conductivity, which demands a conjugate advent of enhancing the power factor. The position of thermoelectrics in the $21^{\text {st }}$ century is subject to the success of this paradigm shift from "phonon glass" to "electron crystal". There is little overlap between lattice thermal conductivity reduction and the power factor improvement, and hence we should pay closer attention to the enhancement approaches with dual purpose.

In this article, we reviewed the favorable electronic band structure and transport parameters for thermoelectric applications as well as the approaches that have been proposed and implemented experimentally, mainly in bulk themoelectrics, to further improve the thermoelectric power factor. With the thermal conductivity of several key thermoelectric materials approaching their minimum theoretical limit, the power factor improvement mechanisms reviewed here can serve as useful tools for further improvement of thermoelectric figure of merit. Among the approaches discussed here, composite engineering has not been explored sufficiently with a focus on power factor enhancement. Experimental implementation of the "invisible doping" idea also remains to 
be seen. Furthermore, theoretical understanding of the recent intriguing experimental results on the power factor enhancement in spark-plasma-sintered Pr: $\mathrm{SrTiO}_{3}$ ceramics [24], and $\mathrm{Bi}$ [257] can open new directions in grain boundary engineering of bulk polycrystalline materials. Perhaps one can appreciate that thermoelectricity effect is a very weak effect when comparing the best power factors obtained so far with the best thermal conductivities available. Even though there are no limits on the thermoelectric power factors, the best observed ones are on the order of 5 $\mathrm{W} / \mathrm{m}-\mathrm{K}$, such a value of power factor is not even close to the conduction ability of a good thermal conductor such as copper $(\mathrm{k}>300 \mathrm{~W} / \mathrm{mK})$. This simple comparison shows that there is a long path a head of thermoelectric society to increase the power factor to larger values!

Several new ideas have been proposed and reported recently which have not been discussed in this review, examples of which are giant thermoelectric effects in topological insulators [258,259] and type-VIII Clathrate $\mathrm{Si}_{46}$ [260], ultracold-atom thermoelectrics [261,262], and highelectron mobility in phonon-glass semimetals [263]. Understanding of such observations and concepts could certainly empower the power factor enhancement toolbox and might result in higher ZT values. Along with the genome project movement initiated by the president, computational materials design and high-throughput materials screenings [264,265] have also been explored recently to discover new potential thermoelectric materials, which have not been discussed in detail here.

In the path to higher performance thermoelectric materials using the approaches discussed in this article, of utmost importance is the reliability and reproducibility of high quality samples. This is imperative for obtaining the correct analysis of the data and the subsequent conclusions being drawn on the improvement mechanisms involved. Just as important is the reliability and reproducibility of the experimental measurements being performed. Characterization of materials before and after densification (e.g. hot-pressing or spark plasma sintering) as well as the measurements of thermoelectric properties using different systems over a broad temperature range (above and below 300K) can significantly help in this regard. Investigation of the microstructure of the samples using different microscopy techniques could also provide crucial information, which would further the understanding of the materials' properties.

In conclusion, thermoelectric research is an exciting inter-disciplinary field across physics, chemistry and materials science and engineering, and the advancement of this field will have direct impacts on the development of these disciplines. It will also provide the students as the next generation of scientists with invaluable skill sets including an intimate knowledge of electron and phonon scattering and the interdependence of the various properties that go into the Figure of Merit, the experimental skills it takes to evaluate and understand these materials including electronic and thermal transport measurements as well as microscopy and chemical analysis techniques, and a broad suite of synthesis techniques to properly synthesize, dope and manipulate the materials (i.e. band gap or degenerate semiconductor). Therefore, one can conclude that thermoelectric materials research is both a daunting task but also a rewarding one. The main reason that we chose to focus a paper on the power factor of thermoelectric materials is that we have approached the limits of the "phonon glass" paradigm in many conventional thermoelectric materials. However, the so-called "playground" of thermoelectric materials research, i.e. the "Periodic Table" is a very large phase space to try to cover. That means there are many opportunities for success but also for failure. We feel that this review article is very 
timely and necessary in understanding the power factor of thermoelectric materials and hope that readers will find it very useful in their future research.

\section{Acknowledgement:}

The work performed in Rutgers is supported by NSF grant 1400246. J. H. would like to acknowledge the support of NSF DMR 1307740. Other work at Clemson University was funded by internal University funds.

\footnotetext{
${ }^{1}$ L. D. Hicks, M. S. Dresselhaus, Phys. Rev. B 47 (1993) 12727.

${ }^{2}$ J. R. Sootsman, D. Y. Chung, M. G. Kanatzidis, Angew. Chem. Int. Ed., 48 (2009) 8616-8639.

${ }^{3}$ G. S. Nolas, D. T. Morelli, T. M. Tritt, Annu. Rev. Mater. Sci. 29 (1999) 89-116.

${ }^{4}$ P. Pichanusakorn, P. Bandaru, Mater. Sci. Eng. R 67 (2010) 19-63.

${ }^{5}$ D. L. Medlin, G. J. Snyder, Curr. Opin. Colloid Interface Sci. 14 (2009) 226-235.

${ }^{6}$ J. P. Heremans, B. Wiendlochaac, A. M. Chamoire, Energy Environ. Sci. 5 (2012) 5510-5530.

${ }^{7}$ H. J. Goldsmid, J. Electron. Mater. 42 (2013) 1482-1489

${ }^{8}$ Y. Pei, H. Wang, G. J. Snyder, Adv. Mater. 24 (2012) 6125-6135.

${ }^{9}$ B. Lenoir, A. Dauscher, M. Cassart, Y. I. Ravich, H. Scherrer, J. Phys. Chem. Solids 59 (1998) 129-134.

${ }^{10}$ J. Androulakis, I. Todorov, J. He, D.-Y. Chung, V. Dravid, M. Kanatzidis, J. Am. Chem. Soc. 133 (2011) 1092010927.

${ }^{11}$ G. Joshi, X. Yan, H. Wang, W. Liu, G. Chen, Z. Ren, Adv. Energy Mater. 1 (2011) 643-647.

12 C. S. Birkel, J. E. Douglas, B. R. Lettiere, G. Seward, N. Verma, Y. Zhang, T. M. Pollock, R. Seshadri, G. D.
} Stucky, Phys.Chem. Chem. Phys. 15 (2013) 6990.

${ }^{13}$ A. F. May, J.-P. Fleurial, G. J. Snyder, Chem. Mater. 22 (2010) 2995.

${ }^{14}$ K. F. Hsu, S. Loo, F. Guo, W. Chen, J. S. Dyck, C. Uher, T. Hogan, E. K. Polychroniadis, M. G. Kanatzidis, Science 303 (2004) 818.

${ }^{15}$ X. Shi, J. Yang, J. R. Salvador, M. Chi, J. Y. Cho, H. Wang, S. Bai, J. Yang, W. Zhang, L. Chen, J. Am. Chem. Soc. 133 (2011) 7837-7846.

${ }^{16}$ X. W. Wang, H. Lee, Y. C. Lan, G. H. Zhu, G. Joshi, D. Z. Wang, J. Yang, A. J. Muto, M. Y. Tang, J. Klatsky, S. Song, M. S. Dresselhaus, G. Chen, Z. F. Ren, Appl. Phys. Lett. 93 (2008) 193121.

${ }^{17}$ M. Ohtaki, T. Tsubota, K. Eguchi, H. Arai, J. Appl. Phys. 79 (1996) 1816.

${ }^{18}$ P. Sun, N. Oeschler, S. Johnsen, B. B. Iversen, F. Steglich, Appl. Phys. Express 2 (2009) 091102.

${ }^{19}$ A. D. LaLonde, Y. Pei, G. J. Snyder, Energy Environ. Sci. 4 (2011) 2090-2096.

${ }^{20}$ L. -D. Zhao, S. -H. Lo, Y. Zhang, H. Sun, G. Tan, C. Uher, C. Wolverton, V. P. Dravid, M. G. Kanatzidis, Nature 508 (2014) 373.

${ }^{21}$ D. M. Rowe, V. L. Kuznetsov, L. A. Kuznetsova, G. Min, J. Phys. D: Appl. Phys. 35 (2002) 2183-2186.

${ }^{22}$ M. Zebarjadi, G. Joshi, G. Zhu, B. Yu, A. Minnich, Y. Lan, X. Wang, M. Dresselhaus, Z. Ren, G. Chen, Nano Lett. 11 (2011) 2225-30.

${ }^{23}$ X. Yan, B. Poudel, Y. Ma, W. S. Liu, G. Joshi, H. Wang, Y. Lan, D. Wang, G. Chen, Z. F. Ren, Nano Lett. 10, 3373 (2010)

${ }^{24}$ A. Mehdizadeh Dehkordi, S. Bhattachary, T. Darroudi, J. W. Graff, U. Schwingenschlögl, H. N. Alshareef, T. M. Tritt, Chem. Mater. 26 (2014) 2478-2485.

${ }^{25}$ W. Liu, X. Tan, K. Yin, H. Liu, X. Tang, J. Shi, Q. Zhang, C. Uher, Phys. Rev. Lett. 108 (2012) 166601.

${ }^{26}$ G. J. Snyder, M. Christensen, E. Nishibori, T. Caillat, B. B. Iversen, Nature Mater. 3 (2004) 458.

${ }^{27}$ D.-Y. Chung, T. Hogan, P. Brazis, M. Rocci-Lane, C. Kannewurf, M. Bastea, C. Uher, M. G. Kanatzidis, Science 287 (2000) 1024.

${ }^{28}$ P. F. Qiu, J. Yang, R. H. Liu, X. Shi, X. Y. Huang, G. J. Snyder, W. Zhang, L. D. Chen, Appl. Phys. Lett. 109 (2011) 063713.

${ }^{29}$ H. Liu, X. Shi, F. Xu, L. Zhang, W. Zhang, L. Chen, Q. Li, C. Uher, T. Day, G. J. Snyder, Nature Mater. 11 (2012) 422.

${ }^{30}$ S. R. Brown, S. M. Kauzlarich, F. Gascoin, G. J. Snyder, Chem. Mater. 18 (2006) 1873-1877.

${ }^{31}$ X. Lu, D. T. Morelli, Y. Xia, F. Zhou, V. Ozolins, H. Chi, X. Zhou, C. Uher, Adv. Energy Mater. 3 (2013) 342.

${ }^{32}$ N. Van Nong, N. Pryds, S. Linderoth, M. Ohtaki, Adv. Mater. 23 (2011) 2484-2490.

${ }^{33}$ Y. Pei, X. Shi, A. LaLonde, H. Wang, L. Chen, G. J. Snyder, Nature 473 (2011) 66. 
34 J. Li, J. Sui, Y. Pei, C. Barreteau, D. Berardan, N. Dragoe, W. Cai, J. He, L.-D. Zhao, Energy Environ. Sci. 5 (2012) 8543.

35 S. R. Culp, J. W. Simonson, S. Joseph Poon, V. Ponnambalam, J. Edwards, T. M. Tritt, Appl. Phys. Lett. 93 (2008) 022105.

36 B. Poudel, Q. Hao, Y. Ma, Y. Lan, A. Minnich, B. Yu, X. Yan, D. Wang, A. Muto, D. Vashaee, X. Chen, J. Liu, M. S. Dresselhaus, G. Chen, Z. Ren, Science 320 (2008) 634-8.

37 J. P. Heremans, V. Jovovic, E. S. Toberer, A. Saramat, K. Kurosaki, A. Charoenphakdee, S. Yamanaka, G. J. Snyder, Science 321 (2008) 554-7.

38 Y. Chen, C. M. Jaworski, Y. B. Gao, H. Wang, T. J. Zhu, G. J. Snyder, J. P. Heremans, X. B. Zhao, New J. Phys. 16 (2014) 013057.

39 N. Mingo, Appl. Phys. Lett. 84 (2004) 2652.

40 T. J. Zhu, K. Xiao, C. Yu, J. J. Shen, S. H. Yang, A. J. Zhou, X. B. Zhao, J. He, J. Appl. Phys. 108 (2010) 044903 .

41 J. M. Ziman, Principles of the Theory of Solids, 2nd ed., Cambridge University Press, Cambridge, 1972.

42 D. J. Singh, in: T. M. Tritt (Ed.), Recent Trends in Thermoelectric Materials Research II, Semiconductors and Semimetals Vol. 70, Academic Press, New York, 2001, p. 125.

43 R. Chasmar, R. Stratton, J. Electron. Control. 7 (1959) 52.

44 G. D. Mahan, J. Appl. Phys. 65 (1989) 1578.

45 J. O. Sofo, G. D. Mahan, Phys. Rev. B 49 (1994) 4565.

46 G.D. Mahan, Good Thermoelectrics, Solid State Physics, Vol. 51, Academic Press, 1997, pp. 82-157.

47 G. S. Nolas, J. Sharp, H. J. Goldsmid, Thermoelectrics: Basic Principles and New Materials Developments, Springer, 2001, p. 292.

48 G. Joshi, H. Lee, Y. Lan, X. Wang, G. Zhu, D. Wang, R. W. Gould, D. C. Cuff, M. Y. Tang, M. S. Dresselhaus, Nano letters 8 (2008) 4670-4.

49 J. R. Sootsman, H. Kong, C. Uher, J. James, D. Angelo, C. Wu, T.P. Hogan, T. Caillat, and M.G. Kanatzidis, Angew. Chem. Int. Ed. 47 (2008) 8618-8622.

50 Makongo, J. P. A.; Misra, D. K.; Zhou, X.; Pant, A.; Shabetai, M. R.; Su, X.; Uher, C.; Stokes, K. L.; Poudeu, P. F. P. J. Am. Chem. Soc. 133 (2011) 18843-52.

51 L. Fu, J. Yang, J. Peng, Q. Jiang, Y. Xiao, Y. Luo, D. Zhang, Z. Zhou, M. Zhang, Y. Cheng and F. Cheng, J. Mater. Chem. A 3 (2015) 1010-1016.

52 Ioffe website: http://www.ioffe.ru/SVA/NSM/Semicond/SiGe/

53 Springer Materials from R. Sehr, L. R. Testardi, J. Phys. Chem. Solids 23 (1962) 1219; B. M. Goltsman et al., Thermodynamic Semiconductor Material Based on Bi2 Te3, US National Technical Information Center, 1973

54 O. Madelung, Semiconductors: Data Handbook. Springer, 2004; H. Sitter, K. Lischka, and H. Heinrich, Phys. Rev. B 16 (1977) 680-687; G. Foley, D. Langenberg, Phys. Rev. B 15 (1977) 4850-4864.

55 S. Öğ̈̈t, K. Rabe, Phys. Rev. B 51 (1995) 10443-10453.

56 H. J. Goldsmid, Electronic Refrigeration, Pion Limited, London, 1986.

57 C. M. Jaworski, J. P. Heremans, Phys. Rev. B 85 (2012) 033204.

58 T. J. Zhu, K. Xiao, C. Yu, J. J. Shen, S.H. Yang, A. J. Zhou, X. B. Zhao, J. He, J. Appl. Phys. 108 (2010) 044903.

59 M. Zou, J.-F. Lia, Takuji Kita, J. Solid State Chem. 198 (2013) 125.

60 C. Fu, T. Zhu, Y. Pei , H. Xie , H. Wang, G. J. Snyder, Y. Liu, Y. Liu, X. Zhao, Adv. Enegry Mater. 4 (2014) 1400600.

61 R. J. Mehta, Y. Zhang, C. Karthik, B. Singh, R. W. Siegel, T. Borca-Tasciuc, G. Ramanath, Nature Mater. 11 (2011) 233-240.

62 C. B. Vining, J. Appl. Phys. 69 (1991) 331.

63 A. J. Minnich, H. Lee, X. W. Wang, G. Joshi, M. S. Dresselhaus, Z. F. Ren, G. Chen, D. Vashaee, Phys. Rev. B 80 (2009) 155327.

64 H. Scherrer, S. Scherrer, in Thermoelectrics Handbook: Macro to Nano, D.M. Rowe (Ed.), CRC Press, New York (2006), p. 27-1.

65 Y. Lan, B. Poudel, Y. Ma, D. Wang, M. S. Dresselhaus, G. Chen, Z. Ren, Nano Lett. 9 (2009) 1419.

66 Y. Pei, A. LaLonde, S. Iwanaga, G. J, Snyder, Energy Environ. Sci. 4 (2011) 2085.

67 K. Biswas, J. He, Q. Zhang, G. Wang, C. Uher, V. P. Dravid, M. G. Kanatzidis, Nature Chem. 3 (2011) 160.

68 T. Caillat, J.-P. Fleurial, A. Borshchevsky, J. Phys. Chem. Solids 58 (1997) 1119-1125. 
69 S. K. Bux, A. Zevalkink, O. Janka, D. Uhl, S. Kauzlarich, J. G. Snyder, J.-P. Fleurial, J. Mater. Chem. A 2 (2014) 215.

70 J. P. Dismukes, L. Ekstrom, E. F. Steigmeier, I. Kudman, D. S. Beers, J. Appl. Phys. 35 (1964) 2899.

71 X. Yan, G. Joshi, W. Liu, Y. Lan, H. Wang, S. Lee, J. W. Simonson, S. J. Poon, T. M. Tritt, G. Chen, Z. F. Ren, Nano Lett. 11 (2011) 556-560.

72 W. J. Xie, J. He, H. J. Kang, X. F. Tang, S. Zhu, M. Laver, S. Y. Wang, J. R. D. Copley, C. M. Brown, Q. J. Zhang and T. M. Tritt, Nano Lett., 10 (2010) 3283.

73 K. Biswas, J. He, I. D. Blum, C.I. Wu, T. P. Hogan, D. N. Seidman, V. P. Dravid and M. G. Kanatzidis, Nature 489 (2012) 414.

74 T. K. Reynolds, J. G. Bales, R. F. Kelley, F. J. DiSalvo, in: M. G. Kanatzidis, T. P. Hogan, S. D. Mahanti (Eds.), Chemistry, Physics and Materials Science of Thermoelectric Materials: Beyond Bismuth Telluride, Kluwer Academic/Plenum, New York, 2003.

75 J. R. Chelikowsky, M. L. Cohen, Phys. Rev. B 14 (1976) 556-582.

76 S. K. Mishra, S. Satpath, O. Jepsen, J. Phys.: Condens. Matter 9 (1997) 461-470.

77 M. G. Kanatzidis, in: T. M. Tritt (Ed.), Recent Trends in Thermoelectric Materials Research I, Semiconductors and Semimetals Vol. 69, Academic, New York, 2001, p. 51.

78 G. A. Slack, in: D. M. Rowe (Ed.), CRC Handbook of Thermoelectrics, CRC Press, London, 1995, p. 407.

79 G. D. Mahan, J. O. Sofo, Proc. Natl. Acad. Sci. USA 93 (1996) 7436-7439.

80 G. D. Mahan, in: T. M. Tritt, M. G. Kanatzidis, H. B. Lyon, Jr., G. D. Mahan (Eds.), Thermoelectric Materials New Directions and Approaches, Materials Research Society Sympos. Proc. 478, 1997, pp. 223-229.

81 S. J. Lee, J. M. Park, P. C. Canfield, D. W. Lynch, Phys. Rev. B 67 (2003) 075104.

82 A. Bentien, S. Johnsen, G. K. H. Madsen, B. B. Iversen, F. Steglich, Europhys. Lett. 80 (207) 17008.

83 J. R. Chelikowsky, M. L. Cohen, Phys. Rev. B 14 (1976) 556.

84 J. Zhou, Z. Sun, X. Cheng, Y. Zhang, Intermetallics 17 (2009) 995.

85 S. J. Lee, J. Zhou, Z. Sun, X. Cheng, Y. Zhang, Phys. Rev. B 67 (2003) 075104

86 J. Zhou, R. Yang, G. Chen, M. S. Dresselhaus, Phys. Rev. Lett. 107 (2011) 226601

87 C. Jeong, M. Lundstrom, J. Electron. Mater. 40 (2011) 738.

88 C. Jeong, R. Kim, M. S. Lundstrom, J. Appl. Phys. 111 (2012) 113707.

89 T. M. Tritt (Ed.), Thermal Conductivity: Theory, Properties, and Applications, Kluwer Academic/Plenum Publishers, New York, 2004.

90 E. S. Toberer, L. L. Baranowski, C. Dames, Annu. Rev. Mater. Res. 42 (2012) 179-209.

91 D. Tuomi, J. Electrochemical Soc. 131(1984) 2319-2325.

92 M. S. Dresselhaus et al., in: M. G. Kanatzidis, T. P. Hogan, S. D. Mahanti (Eds.), Chemistry, Physics and Materials Science of Thermoelectric Materials: Beyond Bismuth Telluride, Kluwer Academic/Plenum, New York, 2003.

93 J. O. Sofo, G. D. Mahan, Appl. Phys. Lett. 65 (1994) 2690.

94 D. A. Broido, T. L. Reinecke, Phys. Rev. B 51 (1995) 13797.

95 T. C. Harman, D. L. Spears, M. j. Manfra, J. Electron. Mater. 25 (1996) 1121.

96 L. D. Hicks, T. C. Harman, X. Sun, M. S. Dresselhaus, Phys. Rev. B 53 (1999) R10493.

97 T. C. Harman, P. J. Taylor, D. L. Spears, M. P. Walsh, J. Electron. Mater. 29 (2000) L1.

98 T. C. Harman, P. J. Taylor, M. P. Walsh, B. E. LaForge, Science 279 (2002) 2229.

99 H. Böttner, G. Chen, R. Venkatasubramanian, MRS Bulletin 31 (2006) 211

100 R. Venkatasubramanian, E. Siivola, B. O'Quinn, in: D. M. Rowe (Ed.), Thermoelectrics Handbook: Macro to Nano, CRC Press, New York, 2006, p. 49-1.

101 D. A. Broido, T. L. Reinecke, in: T. M. Tritt (Ed.), Recent Trends in Thermoelectric Materials Research III, Semiconductors and Semimetals Vol. 71, Academic, New York, 2001, pp. 123-155.

102 A. Shakouri, J. E. Bowers, Appl. Phys. Lett. 71 (1997) 1234.

103 G. D. Mahan, J. Appl. Phys. 76 (1994) 4362.

104 D. M. Rowe, G. Min, Int. Conf. Thermoelectr., 13th Kansas City (1994) pp. 339-42. Piscataway, NJ: IEEE.

105 L. W. Whitlow, T. Hirano, J Appl. Phys. 78 (1995) 5460.

106 D. Vashaee, A. Shakouri, Physical Review Letter 92 (2004) 106103.

107 J. M. O. Zide, D. Vashaee, Z. X. Bian, G. Zeng, J. E. Bowers, A. Shakouri, A. C. Gossard, Phys. Rev. B 74 (2006) 205335.

108 R. Kim, C. Jeong, M. S. Lundstrom, J. Appl. Phys. 107 (2010) 054502.

109 S. V. Faleev, F. Léonard, Phys. Rev. B 77 (2008) 214304. 
110 J. P. Heremans, C. M. Thrush, D. T. Morelli, Phys. Rev. B 70 (2004) 115334.

111 B. Paul, A. Kumar, P. Banerji, J. Appl. Phys. 108 (2010) 064322.

112 D. -K. Ko, Y. Yang, C. B. Murray, ACS Nano 11 (2011) 2841.

113 S. N. Zhang, T. J. Zhu, S. H. Yang, C. Yu, X. B. Zhao, J. Alloys Compd. 499 (2010) 215.

114 Z. Xiong, X. Chen, X. Y. Huang, S. Q. Bai, L. D. Chen, Acta Mater. 58 (2010) 3995.

115 A. Soni, Y. Shen, M. Yin, Y. Zhao, L. Yu, X. Hu, Z. Dong, K. A. Khor, M. S. Dresselhaus, Q. Xiong, Nano Lett. 12 (2012) 4305.

116 M. He, J. Ge, Z. Lim, X. Feng, X. Wang, H. Lu, Y. Yang, F. Qiu, Energy Environ. Sci. 5 (2012) 8351.

117 S. I. Kim, K. Ahn, D.-H. Yeon, S. Hwang, H.-S. Kim, S. M. Lee, K. H. Lee, Appl. Phys. Express 4 (2011) 091801.

118 Y. C. Dou, X. Y. Qin, D. Li, L. L. Li, T. H. Zhou, Q. Q. Wang, J. Appl. Phys. 114 (2013) 044906.

119 Y. Wang, X. Zhang, L. Shen, N. Bao, C. Wan, N.-H. Park, K. Koumoto, A. Gupta, J. Power Sources 241 (2013) 255.

120 N. Neophytou, X. Zianni, H. Kosina, S. Frabboni, B. Lorenzi, D. Narducci, Nanotech. 24 (2013) 205402.

121 T. H. Zou, X. Y. Qin, D. Li, B. J. Ren, G. L. Sun, Y. C. Dou, Y. Y. Li, L. L. Li, J. Zhang, H. X. Xin, J. Appl. Phys. 115 (2014) 053710.

122 Y. Zhang, J. H. Bahk, J. Lee, C. S. Birkel, M. L. Snedaker, D. Liu, H. Zhang, M. Moskovits, A. Shakouri, G. Stucky, Adv. Mater. 26 (2014) 2755.

123 W. J. Xie, Y. G. Yan, S. Zhu, M. Zhou, S. Populoh, K. Galazka, S. J. poon, A. Weidenkaff, J. He, X. F. Tang, T. M. Tritt, Acta Mater. 61 (2013) 2087.

124 M. Liu, X. Y. Qin, Appl. Phys. Lett. 101 (2012) 132103.

125 J. H. Bahk, Z. Bian, A. Shakouri, Phys. Rev. B 89 (2014) 075204.

126 Y. I. Ravich, B. A. Efimova, V. I. Tamarchenko, Physica Status Solidi (B) 43 (1971) 433.

127 Y. I. Ravich, in: D. M. Rowe (Ed.), CRC Handbook of Thermoelectrics, CRC Press, New York, 1995, pp. 6773.

128 A. Blandin, J. Friedel, Le Journal de Physique et le Radium 20 (1959) 160.

129 J. J. Sakurai, Modern Quantum Mechanics, Section 7.8, revised ed., Prentice Hall Inc., 1994.

130 E. P. Wigner, Phys. Rev. 98 (1955) 145.

131 E. Daniel and J. Friedel in: J.G. Daunt et al., (Eds.), Low-Temperature Physics LT9, Plenum Press, New York, 1965, pp. 933-954.

132 J. P. Heremans, in: D. M. Rowe (Ed.), Thermoelectrics and Its Energy Harvesting: Materials, Preparation, and Characterization in Thermoelectrics, CRC Press, Boca Raton, FL, 2012, p. 12-1.

133 J. D. König, M. D. Nielsen, Y. Gao, M. Winkler, A. Jacquot, H. Böttner, J. P. Heremans, Phys. Rev. B 84 (2011) 205126.

134 P. W. Anderson, Theory of Superconductivity in the High-Tc Cuprates, Princeton University Press, Princeton, 1997.

135 C. M. Jaworski, B. Wiendlocha, V. Jovovic, J. P. Heremans, Energy Environ. Sci. 4 (2011) 4155.

136 C. M. Jaworski, V. Kulbachinskii, J. P. Heremans, Physical Review B 80 (2009) 233201- 233204.

137 Q. Zhang, H. Wang, W. Liu, H. Wang, B. Yu, Q. Zhang, Z. Tian, G. Ni, S. Lee, K. Esfarjani, G. Chen, Z. Ren, Energy Environ. Sci. 5 (2012) 5246.

138 M. K. Zhitinskaya, S. A. Nemov, T. E. Svechnikova, Phys. Solid State 40 (1998) 1297.

139 V. Jovovic, S. J. Thiagarajan, J. P. Heremans, T. Komissarova, D. R. Khoklov, A. Nicorici, J. Appl. Phys. 103 (2008) 053710.

140 K. Ahn, M. Han, J. He, J. Androulakis, S. Ballikaya, C. Uher, V. P. Dravid, M. G. Kanatzidis, J. Am. Chem. Soc. 132 (2010) 5227.

141 P. K. Rawat, B. Paul, P. Banerji, ACS Appl. Mater. Interfaces 6 (2014) 3995.

142 L. Pfeiffer, K. West, Physica E 20 (2003) 57-64.

143 B. Yu, M. Zebarjadi, H. Wang, K. Lukas, H. Wang, D. Wang, C. Opeil, M. Dresselhaus, G. Chen, Z. Ren, Nano Lett. 12 (2012) 2077-82.

144 D. Wu, Y. Pei, Z. Wang , H. Wu , L. Huang , L.-D. Zhao, J. He, Adv. Func. Mater. 24 (2014) 7763-7771.

145 Q. R. Hou, B. F. Gu, Y. B. Chen, Y. J. He, Physica Status Solidi (a) 209 (2012) 1307-1312.

146 Q. R. Hou, B. F. Gu, Y. B. Chen, Y. J. He, J. L. Sun, Appl. Phys. A 114 (2013) 943-949.

147 W. J. Liang, A. I. Hochbaum, M. Fardy, O. Rabin, M. J. Zhang, P. D. Yang, Nano Lett., 9 (2009) 1689.

148 B. M. Curtin, E. A. Codecido, S. Kramer, J. E. Bowers, Nano Lett. 13 (2013) 5503.

149 T. Caillat, M. Carle, P. Pierrat, H. Scherrer, S. Scherrer, J. Phys. Chem. Solids 53 (1992) 1121 
150 M. Carle, P. Pierrat, C. Lahalle-Gravier, S. Scherrer, H. Scherrer, J. Phys. Chem. Solids 56 (1995) 201

151 V. K. Zaitsev, in CRC Handbook of Thermoelectrics, D.M. Rowe (Ed.), CRC Press, New York (1995), p. 299

152 M. I. Fedorov, V. K. Zaitsev, in: D.M. Rowe (Ed.), CRC Handbook of Thermoelectrics, CRC Press, New York, 2006, p. 299

153 I. Terasaki, Y. Sasago and K. Uchinokura, Phys. Rev. B 56 (1997) R12685(R).

154 B. C. Sales, R. Jin, K. A. Affholter, P. Khalifah, G. M. Veith, D. Mandrus, Phys. Rev. B 70 (204) 174419.

155 S. Bhattacharya, D. K. Aswal, A. Singh, C. Thinaharan, N. Kulkarni, S. K. Gupta, J. V. Yakhmi, J. Cryst. Growth 277 (2005) 246-251.

156 G. D. Tang, H. H. Guo, T. Yang, D. W. Zhang, X. N. Xu, L. Y. Wang, Z. H. Wang, H. H. Wen, Z. D. Zhang, Y. W. Du, Appl. Phys, Lett. 98 (2011) 202109.

157 F. K. Lotgering, J. Inorg. Nucl. Chem. 9 (1959) 113-123.

158 O. Ben-Yehuda, Y. Gelbstein, Z. Dashevsky, R. Shuker, M. P. Dariel, Proc. 25th Int'1 Conf. Thermoelectrics ICT06, (2006) 492.

159 O. Ben-Yehuda, R. Shuker, Y. Gelbstein, Z. Dashevsky, M. P. Dariel, J. Appl. Phys. 101 (2007) 113707.

160 J. J. Shen, L. P. Hu, T. J. Zhu, X. B. Zhao, Appl. Phys. Lett. 99 (2011) 124102.

161 J. Seo, C. Lee, K. Park, Mater. Sci. Eng. B 54 (1998) 135.

162 J. Seo, K. Park, C. Lee, Mater. Res. Bull. 33 (1998) 553.

163 L. D. Ivanova, L. I. Petrova, Yu. V. Granatkina, V. S. Zemskov, O. B. Sokolov, S. Ya. Skipidarov, N. I. Duvankov, Inorg. Mater. 44 (2008), 687.

164 V. M. Segal, Mater. Sci. Eng. A 386 (2004) 269.

165 Z. -C. Chen, K. Suzuki, S. Miura, K. Nishimura, K. Ikeda, Mater. Sci. Eng. A 500 (2009) 70.

166 J.-T. Im, K. T. Hartwig, J. Sharp, Acta Materialia 52 (2004) 49.

167 X. A. Fan, J. Y. Yang, W. Zhu, S. Q. Bao, X. K. Duan, C. J. Xiao, K. Li, J. Alloys Compd. 461 (2008) 9-13.

168 S. S. Kim, S. Yamamoto, T. Aizawa, J. Alloys Compd. 375 (2004) 107.

169 T. Hayashi, M. Sekine, J. Suzuki, Y. Horio, H. Takizawa, Mater. Trans. 48(2007) 2724.

170 A. Mehdizadeh Dehkordi, D. Vashaee, Physica Status Solidi A 209 (2012) 2131.

171 J. J. Shen,1 Z. Z. Yin, S. H. Yang, C. Yu, T. J. Zhu, X. B. Zhao, J. Electron. Mater. 40 (2011) 1095.

172 T. Zhu, Z. Xu, J. He, J. Shen, S. Zhu, L. Hu, T. M. Tritt, X. Zhao, J. Mater. Chem. A 1 (2013) 11589.

173 S. Grasso, N. Tsujii, Q. Jiang, J. Khaliq, S. Maruyama, M. Mirand, K. Simpson, T. Mori, M. J. Reece, J. Mater. Chem. C 1 (2013) 2362

174 Q. Jiang, H. Yan, J. Khaliq, H. Ning, S. Grasso, K. Simpson, M. J. Reece, J. Mater. Chem. A 2 (2014) 5785.

175 J. Navrátil, Z. Starý, T. Plechác $\square$ ek, Mater. Res. Bull. 31 (1996) 1559.

176 L. Hu, H. Gao, X. Liu, H. Xie, J. Shen, T. Zhu, X. Zhao, J. Mater. Chem. 22 (2012) 16484.

177 L. Hu, T. Zhu, X. Liu, X. Zhao, Adv. Funct. Mater. 24 (2014) 5211.

178 L. P. Hu, X. H. Liu, H. H. Xie, J. J. Shen, T. J. Zhu, X. B. Zhao, Acta Materialia 60 (2012) 4431-4437.

179 S. D. Bhame, D. Pravarthana, W. Prellier, J. G. Noudem, Appl. Phys. Lett. 102 (2013) 211901.

180 M. Mikami, E. Guilmeau, R. Funahashi, K. Chong, D. Chateigner, J. Mater. Res. 20 (2005) 2491.

181 M. Prevel, S. Lemonnier, Y. Klein, S. Hébert, D. Chateigner, B. Ouladdiaf, J. G. Noudem, J. Appl. Phys. 98 (2005) 093706.

182 Y.-H. Lin, J. Lan, Z. Shen, Y. Liu, C.-W. Nan, J.-F. Li, Appl. Phys. Lett. 94 (2009) 072107.

183 A. C. Masset, C. Michel, A. Maignan, M. Hervieu, O. Toulemonde, F. Studer, B. Raveau, J. Hejtmanek, Phys. Rev. B 62 (2000) 166.

184 S. Horii, I. Matsubara, M. Sano, K. Fujie, M. Suzuki, R. Funahashi, M. Shikano, W. Shin, N. Murayama, J.-I. Shimoyama, K. Kishio, Jpn. J. Appl. Phys. 42 (2003) 7018-7022.

185 Y. Zhou, I. Matsubara, S. Horii, T. Takeuchi, R. Funahashi, M. Shikano, J.-I. Shimoyama, K. Kishio, W. Shin, N. Izu, N. Murayama, J. Appl. Phys. 93 (2003) 2653.

186 Y. Huang, B. Zhao, J. Fang, R. Ang, Y. Sun, J. Appl. Phys. 110 (2011) 123713.

187 T. Kuribayashi, M.-G. Sung, T. Itoh, K. Sassa, S. Asai, Mater. Trans. 47 (2006) 2387-2392.

188 H. Kaga, Y. Kinemuchi, K. Watari, S. Tanaka, A. Makiya, Z. Kato, K. Uematsu, J. Mater. Res. 22 (2007) 2917.

189 T. Tani, J. Ceram. Soc. Jpn. 114 (2006) 363-370.

190 S. Tajima, T. Tani, S. Isobe, K. Koumoto, Mater. Sci. Eng. B 86 (2001) 20-25.

191 T. Tani, H. Itahara, C. Xia, J. Sugiyama, J. Mater. Chem. 13 (2003) 1865-1867.

192 N. A. Heinz, T. Ikeda , Y. Pei , G. J. Snyder, Adv. Funct. Mater. 24 (2014) 2135-2153.

193 C. Herring, J. Appl. Phys. 31 (1960) 1939-1952.

194 I. Webman, J. Jortner, Phys. Rev. B 16 (1977) 2959-2964. 
195 J. P. Straley, J. Phys. D: Appl. Phys. 14 (1981) 2101-2105.

196 D. J. Bergman, O. Levy, J. Appl. Phys. 70 (1991) 6821.

197 D. J. Bergman, L. G. Fel, J. Appl. Phys. 85 (1999) 8205.

198 J. P. Heremans, C. M. Jaworski, Appl. Phys. Lett. 93 (2008) 122107.

199 D. Vashaee, A. Shakouri, Phy. Rev. Lett. 92 (2004) 106103.

200 B. Moyzhes, V. Nemchinsky, Appl. Phys. Lett. 73 (1998) 1895.

201 D. A. Broido, N. Mingo, Phys. Rev. B 74 (2006) 195325.

202 N. Neophytou, X. Zianni, H. Kosina, S. Frabboni, B. Lorenzi, D. Narducci, J. Electron. Mater. 43 (2014) 1896.

203 Z. Bian, A. Shakouri, Appl. Phys. Lett. 89 (2006) 212101.

204 Z. Bian, H. Wang, Q. Zhou, A. Shakouri, Phys. Rev. B 75 (2007) 245208.

205 G. D. Mahan, J. Appl. Phys. 70 (1991) 4551.

206 Y. Yang, F. Y. Ma, C. H. Lei, Y. Y. Liu, J. Y. Li, Appl. Phys. Lett. 102 (2013) 053905.

207 Z. Zamanipour, D. Vashaee, J. Appl. Phys. 112 (2012) 093714.

208 J. Peng, L. Fu, Q. Liu, M. Liu, J. Yang, D. Hitchcock, M. Zhou, Jian He, J. Mater. Chem. A 2 (2014) 73-79.

209 B. Du, H. Li, J. Xu, X. Tang, C. Uher, Chem. Mater. 22 (2010) 5521-5527.

210 Y. Wang, X. Zhang, L. Shen, N. Bao, C. Wan, N.-H. Park, K. Koumoto, A. Gupta, J. Power Sources 241 (2013) 255-258.

211 X. Zhao, H. Wang, S. Wang, D. Elhadj, J. Wangab, G. Fu, RSC Adv. 4 (2014) 57148.

212 A. Mehdizadeh Dehkordi, S. Bhattacharya, J. He, H. N. Alshareef, T. M. Tritt, Appl. Phys. Lett. 104 (2014) 193902.

213 H. J. Wu, L.-D. Zhao, F. S. Zheng, D. Wu, Y. L. Pei, X. Tong, M. G. Kanatzidis, J. Q. He, Nature Commun. 5 (2014).

214 B. Zhang, J. Sun, H. E. Katz, F. Fang, R. L. Opila, ACS Appl. Mater. Interfaces 2 (2010) 3170-3178.

215 C. Yu, K. Choi, L. Yin, J. C. Grunlan, ACS Nano 5 (2011) 7885-7892.

216 S. K. Yee, N. E. Coates, A. Majumdar, J. J. Urban, R. A. Segalman, Phys. Chem. Chem. Phys, 15 (2013) 40244032.

217 T. Koga, X. Sun, S. B. Cronin, M. S. Dresselhaus, Appl. Phys. Lett. 73 (1998) 2950.

218 T. Koga, Ph.D. Thesis, Harvard University (2000), pp. 192-198.

219 M. S. Dresselhaus, Y.-M. Lin, S. B. Cronin, O. Rabin, M. R. Black, G. Dresselhaus, in: T. M. Tritt (Ed.), Recent Trends in Thermoelectric Materials Research III, Semiconductors and Semimetals Vol. 71, New York, 2001, pp. 1-121.

220 T. Koga X. Sun, S. B. Cronin, M. S. Dresselhaus, Appl. Phys. Lett. 75 (1999) 2438.

221 T. Koga, X. Sun, S. B. Cronin, M. S. Dresselhaus, in: The 18th International Conference on Thermoelectrics: ICT Symposium Proceedings, Baltimore, 1999, p. 378.

222 T. Koga, S. B. Cronin, M. S. Dresselhaus, J. L. Liu, K. L. Wang, Appl. Phys. Lett. 77 (2000) 1490.

223 O. Rabin, Y.-M. Lin, M. S. Dresselhaus, Appl. Phys. Lett. 79 (2001) 81.

224 T. Koga, M. S. Dresselhaus, X. Sun, S. B. Cronin, Superlattice Structures Having Selected Carrier Pockets and Related Methods, US Patent 6,627,809 B1, 2003.

225 A. D. LaLonde, Y. Pei, H. Wang, G. J. Snyder, Mater. Today 14 (2011) 526.

226 Y. Pei, A. D. LaLonde, N. A. Heinz, X. Shi, S. Iwanaga, H. Wang, L. Chen, G. J. Snyder, Adv. Mater. 23 (2011) 5674-5678.

227 Y. Pei, A. D. LaLonde, A. N. Heinz, G. J. Snyder, Adv. Energy Mater. 2 (2012) 670-675.

228 Y. Pei, H. Wang, Z. M. Gibbs, A. D. LaLonde,G. J. Snyder, NPG Asia Mater. 4 (2012) 1-6.

229 L. D. Zhao, H. J. Wu, S. Q. Hao, C. I. Wu, X. Y. Zhou, K. Biswas, J. Q. He, T. P. Hogan, C. Uher, C. Wolverton, V. P. Dravid, M. G. Kanatzidis, Energy Environ. Sci. 6 (2013) 3346.

230 S. A. Saleh, Phil. Mag. 94 (2014) 3183.

231 V. K. Zaitsev, M. I. Fedorov, E. A. Gurieva, I. S. Eremin, P. P. Konstantinov, A.Y. Samunin, M.V. Vedernikov, Proc. 24th Int'l Conf. Thermoelectrics ICT05, (2005) 189.

232 V. K. Zaitsev, M. I. Fedorov, E. A. Gurieva, I. S. Eremin, P. P. Konstantinov, A. Y. Samunin, M. V.

Vedernikov, Phys. Rev. B 74 (2006) 045207.

233 G. Tan, L.-D. Zhao, F. Shi, J. W. Doak, S.-H. Lo, H. Sun, C. Wolverton, V. P. Dravid, C. Uher, M. G. Kanatzidis, J. Am. Chem. Soc. 136 (2014) 7006-7017.

234 A. Banik, K. Biswas, J. Mater. Chem. A, 2 (2014) 9620-9625.

235 G. Tan, F. Shi, J. W. Doak, H. Sun, L.-D. Zhao, P. Wang, C. Uher, C. Wolverton, V. P. Dravid, M. G. Kanatzidis, Energy Environ. Sci. 8 (2015) 267. 
236 C. Ramsauer, Annalen der Physik 4 (1921) 513-540.

237 V. A. Bailey, J. S. Townsend, Philos. Mag. S. 6, 42 (1921) 873-891.

238 M. Zebarjadi, B. Liao, K. Esfarjani, M. Dresselhaus, G. Chen, Adv. Mater. 25 (2013) 1577.

239 A. Alu, N. Engheta, Optics Express 15 (2007) 7578.

240 A. Alù, N. Engheta, Phys. Rev. E 72 (2005) 016623.

241 W. Cai, U. K. Chettiar, A. V. Kildishev, V. M. Shalaev, Nature Photonics 1 (2007) 224-227.

242 B. Liao, M. Zebarjadi, K. Esfarjani, G. Chen, Phys. Rev. Lett. 109 (2012) 126806.

243 R. Fleury, A. Alù, Phys. Rev. B 87 (2013) 045423.

244 W. Shen, T. Tian, B. Liao, M. Zebarjadi, Phys. Rev. B 90 (2014) 075301.

245 J. He, T. M. Tritt, in: J. Garcia-Martinez, J. (Ed.), Nanotechnology for the Energy Challenge, Wiley-VCH, Weinhaim, Germany, 2010. pp 47-78.

246 C. J. Vineis, A. Shakouri, A. Majumdar, M. G. Kanatzidis, Adv. Mater. 22 (2010) 3970-3980.

247 M. Kanatzidis, Chem. Mater. 22 (2010) 648.

248 W. S. Liu, X. Yan, G. Chen, Z. F. Ren, Nano Energy 1 (2012) 42.

249 J. W. Sharp, S. J. Poon, H. J. Goldsmid, Phys. Status Solidi (a) 187 (2001) 507.

250 X. Ji, B. Zhang, Z. Su, T. Holgate, J. He, T. M. Tritt, Physica Status Solidi (a) 206 (2009) 221.

251 J. Lim, K. Hippalgaonkar, S. C. Andrews, A. Marjumdar, P. D. Yang, Nano Lett. 12 (2012) 2475 and the reference therein.

252 X. Ji, J. He, Z. Su, N. Gothard, T. M. Tritt, J. Appl. Phys. 104 (2008) 034907.

253 B. Zhang, J. He, X. Ji, T. M. Tritt, A. Kumbhar, Appl. Phys. Lett. 89 (2006)163114.

254P. N. Alboni, X. Ji, J. He, N. Gothard, T. M. Tritt. J. Appl. Phys. 103 (2008) 113707.

255 For example, M. S. Dresselhaus, G. Chen, M. Y. Tang, R. G. Yang, H. Lee, D. Wang, Z. F. Ren, J. P. Fleurial, P. Gogna, Adv. Mater. 19 (2007) 1043.

256 P. Puneet, R. Podila, M. Karakaya, S. Zhu, J. He, T. M. Tritt, M. S. Dresselhaus, A. M. Rao, Sci. Rep. 3 (2013) 3212.

257 P. Puneet, R. Podila, S. Zhu, M. J. Skove, T. M. Tritt, J. He, A. M. Rao, Adv. Mater. 25 (2013) 1033.

258 P. H. Chang, M. S. Bahramy, N. Nagaosa, B. K. Nikolic, Nano Lett. 14 (2014) 3779-3784.

259 Y. Xu, Z. Gan, S.-C. Zhang, Phys. Rev. Lett. 112 (2014) 226801

260 P. Norouzzadeh, C. W. Myles, D. Vashaee, Sci. Reports 4 (2014) 1.

261 J.-P. Brantut, C. Grenier, J. Meineke, D. Stadler, S. Krinner, C. Kollath, T. Esslinger, A. Georges, Science 342 (2013) 713.

262 T. T. Heikkilä, Science 342 (2013) 703.

263 S. Ishiwata, Y. Shiomi, J. S. Lee, M. S. Bahramy, T. Suzuki, M. Uchida, R. Arita, Y. Taguchi, Y. Tokura, Nature Mater. 12 (2013) 512.

264 S. Curtarolo, G. L. W. Hart, M. Buongiorno Nardelli, N. Mingo, S. Sanvito, O. Levy, Nature Mater. 12 (2013) 191.

265 J. Carrete, N. Mingo, S. Wang, S. Curtarolo, Adv. Func. Mater. 24 (2014) 7427. 